\title{
5. Die gruppenspezifische Arbeitskräftelenkung
}

\section{Flüchtlinge und Vertriebene}

Bereits Ende der vierziger Jahre hatte sich abgezeichnet, daß gezielte arbeitsmarktpolitische Programme für die "Umsiedler" bis auf wenige Ausnahmen ${ }^{1}$ nicht vorgesehen waren. Aus sicherheits- aber auch aus gesamtwirtschaftlichen Erwägungen heraus verzichteten SED-Führung und DWK auf solche Maßnahmen zugunsten einer Bevölkerungsgruppe. Flüchtlinge und Vertriebene waren für die Arbeitsverwaltung nicht wegen ihrer Herkunft interessant, sondern - wie die Gesamtbevölkerung überhaupt - im Hinblick auf ihre Arbeitsfähigkeit. Die Arbeitsmarktpolitik zielte insgesamt auf eine stetige Erhöhung der Erwerbstätigenzahlen, so daß die Eingliederung der "Umsiedler" in die Teilarbeitsmärkte diesem Ziel untergeordnet wurde. Von ausschlaggebender Bedeutung war in der sich verschärfenden Systemauseinandersetzung zwischen Ost und West, und damit zwischen der DDR und der Bundesrepublik, die Beseitigung der Arbeitslosigkeit. Unter den in der SBZ bzw. der frühen DDR registrierten Arbeitslosen stellten weibliche "Umsiedler" einen überproportional hohen Anteil². Da Flüchtlinge und Vertriebene ab Frühjahr 1949 in der Arbeitsmarktstatistik nicht mehr separat ausgewiesen wurden, ist im übrigen eine Analyse des weiteren Integrationsverlaufs nicht möglich. Sowohl die Landesverwaltungen als auch das Ministerium für Arbeit wandten sich dagegen, die statistische Erhebung über die Zahl der erwerbslosen „Umsiedler" fortzuführen oder sogar zu verfeinern. So betonte etwa das brandenburgische Ministerium für Arbeit und Sozialwesen, daß „das Problem der arbeitslosen Umsiedler nicht durch Erweiterung einer Statistik gelöst wird“3. Die Landesverwaltungen riefen zwar in unregelmäßigen Abständen dazu auf, arbeitsuchende Vertriebene bevorzugt zu vermitteln'; darüber entschied aber letztlich die konkrete Arbeitsmarktsituation in den Städten und Gemeinden. Des weiteren verband sich für die „Umsiedler“ die Arbeitsplatzsuche mit der Wohn-

1 Dazu zählte vor allem das Kunsthandwerk in Thüringen. Vgl. Kapitel I.6.

2 BAB, DQ 2/906, Bl. 245, Staatssekretär Peschke am 3. 12. 1949 an alle Landesregierungen. Der Anteil der Arbeitslosen unter den registrierten „Umsiedlern“ lag auch Anfang der fünfziger Jahre noch über dem der eingesessenen Bevölkerung. Zum 31.1. 1950 meldete Thüringen eine durchschnittliche Arbeitslosenquote von 3,71\%; dieser Anteil lag dagegen bei den Vertriebenen bei 6,35\%. Vgl. BAB, DQ 2/1726, Arbeitsplan des thüringischen Ministeriums für Arbeit und Sozialwesen (HA Arbeit und Sozialfürsorge) vom 24. 6. 1950 für das II. Halbjahr 1950, S. 5.

3 BAB, DQ 2/1245, Ministerium für Arbeit und Sozialwesen der Landesregierung Brandenburg am 6. 12. 1949 an das Ministerium für Arbeit und Gesundheitswesen.

4 Vgl. BAB, DQ 2/1726, Arbeitsplan des thüringischen Ministeriums für Arbeit und Sozialwesen (HA Arbeit und Sozialfürsorge) vom 24.6. 1950 für das II. Halbjahr 1950, S. 5. Die LDP schlug im Sommer 1950 vor, Erleichterungen für die Rückkehr der "Umsiedler“ in ihre früheren Berufe zu schaffen. So sollte beispielsweise die Zulassung und Gründung von Gewerbebetrieben ehemals Selbständiger erleichtert und gefördert werden. ADL, LDPD, 2095, Vorschläge der LDP vom 8. 8. 1950 zum Fünfjahrplan, S. 47. Generell galt, daß Flüchtlinge und Vertriebene bei der Arbeitsplatzsuche flexibel sein mußten und oftmals eine Beschäftigung zu akzeptieren hatten, die ihrer Ausbildung und ihrem bisherigen Berufsweg nicht entsprach. Das Sekretariat des ZK beauftragte Ende September 1950 die Landesregierung Mecklenburg, „Umsiedler, soweit sie arbeitsfähig sind, in den Produktionsprozeß einzugliedern und ihnen den Umzug an den Arbeitsplatz zu ermöglichen.“ SAPMO, DY $30 / J$ IV 2/3/141, Bl. 7. Da der Beschluß allerdings mit keinen Auflagen oder weiteren ergänzenden Anlagen versehen war, handelte es sich hierbei nur um eine unverbindliche Absichtserklärung. 
raumsuche; letzteres stellte mitunter ein Hindernis für die Seßhaftwerdung der Neuankömmlinge insgesamt dar und erschwerte die berufliche Integration zusätzlich. Schließlich darf nicht übersehen werden, daß Flüchtlinge und Vertriebene bei der Arbeitsplatzsuche mit der eingesessenen Bevölkerung um ein knappes Gut konkurrierten.

Auf die Bedeutung der Binnenwanderung für die Integration der Flüchtlinge und Vertriebenen wurde bereits im vorherigen Kapitel hingewiesen. Dieser Prozeß war mit der DDR-Gründung keineswegs abgeschlossen. Zu diesem Zeitpunkt begannen die Arbeitsämter das Bestreben zahlreicher „Umsiedler“ zu registrieren, in die Bundesrepublik abzuwandern 5 . Somit trat zur Binnenwanderung auch noch das Phänomen der Westflucht hinzu, das nunmehr immer bedeutsamer werden sollte und auch die Arbeitsverwaltung zunehmend in den Bann zog, da sich in dieser Gruppe ein nicht unbeträchtlicher Anteil Jugendlicher und Facharbeiter befand. Die Arbeitsämter wurden aufgefordert, eine detaillierte Erhebung des Facharbeiterbedarfs durchzuführen, um eine bedarfsgerechte Steuerung der Arbeitskräfte zu garantieren. Auf diese Weise sollte der Gefahr einer Abwanderung von qualifizierten Arbeitern begegnet werden. Anfang der fünfziger Jahre gab es außerdem noch vereinzelte „Umsiedler“-Transporte aus Polen, unter denen sich offensichtlich eine größere Anzahl von Bergarbeitern befand, die direkt in den Steinkohlenbergbau bei Zwickau-Oelsnitz oder zum Mansfelder Schieferbergbau weitergeleitet werden sollten 6 . Solche Transporte blieben zwar die Ausnahme und stellten an die Arbeitsverwaltung nicht mehr dieselben Anforderungen wie in den ersten Nachkriegsjahren, als Tausende von Flüchtlingen mit Wohnraum und mit Arbeit versorgt werden mußten. Dabei kam es aber nach wie vor zu zahlreichen Fehlvermittlungen ${ }^{7}$, d.h. berufsfremdem Einsatz von „Umsiedlern“, welche die zuständigen Arbeitsämter nachträglich korrigieren mußten8.

Obwohl die Flucht- und Wanderungsbewegung in den Westen Deutschlands unmittelbar nach Gründung der DDR eingesetzt hatte9, blieb die Anzahl der "Umsiedler" und deren Anteil an der Gesamtbevölkerung zunächst nahezu konstant. Mitte 1953 registrierte das Staatssekretariat für Innere Angelegenheiten rund 4312000 Vertriebene, was einem Bevölkerungsanteil von 24,2 Prozent entsprach $^{10}$. Rückblickend wurde besonders die Eingliederung in die öffentliche Ver-

5 Im Arbeitsministerium kursierten wilde Spekulationen: Einzelne Mitarbeiter gingen davon aus, daß etwa 70\% der Umsiedler den Wunsch äußern würden, in die Bundesrepublik zu ziehen. BAB, DQ 2/1720, Aktenvermerk Walters vom 4. 5. 1951. Nach Angaben Meinickes befanden sich unter den mindestens 2,7 Millionen Menschen, die bis zum Mauerbau 1961 die DDR verließen, etwa 950000 Vertriebene. Meinicke, Flüchtlinge, S. 79. Bei Heidemeyer liegen die Zahlenangaben - auf der Basis der Volkszählung 1961 - niedriger. Vgl. Heidemeyer, Flucht und Zuwanderung, S. 44. Vgl. zuletzt: Heidemeyer, Vertriebene als Sowjetzonenflüchtlinge.

6 BAB, DQ 2/1720, IG Bergbau, Zentralvorstand Halle, am 3. 3. 1950 an den FDGB-Bundesvorstand (Abt. Sozialpolitik).

7 Diese beschränkten sich im wesentlichen auf Sachsen-Anhalt. Vgl. BAB, DQ 2/1720, Aktenvermerk (o.Verf.) vom 1. 8. 1951.

8 Vgl. LA Magdeburg LHA, Rep. K MW, Nr. 9448, Ministerium für Arbeit (Abt. Arbeitskraftlenkung) am 1.8. 1951 an das Ministerium für Wirtschaft (Abt. Arbeitskraftlenkung) von SachsenAnhalt.

9 Vgl. dazu die Zahlenangaben bei Heidemeyer, Flucht und Zuwanderung, S. 44.

$10 \mathrm{BAB}, \mathrm{DQ} 2 / 49, \mathrm{Bl} .135 \mathrm{f}$., hier Bl. 135, Notiz des Staatssekretariats für Innere Angelegenheiten (Abt. Bevölkerungspolitik) vom 30.7.1953. 
waltung hervorgehoben: So waren am 31. März 1949 insgesamt 72865 „Umsiedler" in der Verwaltung beschäftigt, 8734 bei der Post, 35350 bei der Reichsbahn sowie 23411 als Lehrer ${ }^{11}$. Eine systematische Erhebung war allerdings nicht durchgeführt worden; vielmehr handelte es sich nur um Einzelergebnisse. Auch wenn in den Berichten der Innenverwaltung partiell Kritik am Integrationsverlauf geübt wurde bzw. Hürden benannt wurden ${ }^{12}$, blieb doch das Gesamtergebnis eindeutig: Aus Sicht der DDR-Regierung und der SED-Führung war die Eingliederung der Flüchtlinge und Vertriebenen in der DDR bereits zu Beginn der fünfziger Jahre erfolgreich abgeschlossen worden. Unterstützende Sondermaßnahmen und eine weitere kontinuierliche Untersuchung dieses keineswegs reibungslos verlaufenden Prozesses wurden jedoch nicht mehr für notwendig erachtet.

\section{Frauen}

Obwohl die Arbeitsverwaltung frühzeitig die Einbeziehung von erwerbsfähigen Frauen in den Produktionsprozeß propagierte, verhinderten zahlreiche retardierende Faktoren die reibungslose Umsetzung dieses Ziels. Auf die vergleichsweise hohe Beschäftigungslosigkeit unter den Frauen ist bereits aufmerksam gemacht worden. Auch hierbei gab es unterschiedliche Entwicklungen in den einzelnen Ländern: So meldete das sächsische Ministerium für Arbeit und Sozialwesen ein stetiges Ansteigen der Arbeitslosenquote von 40 Prozent (Dezember 1947) über 55 Prozent (Dezember 1948) auf 75 Prozent (Dezember 1949)13. Als Ursachen für diese Entwicklung wurden der Niedergang der Textil- und Bekleidungsindustrie mit seinem hohen Anteil an weiblichen Arbeitskräften sowie der überproportional hohe Anteil von ungelernten Hilfskräften unter den Frauen genannt. Diese Beschäftigtengruppen seien in Sachsen am stärksten von der Entlassungswelle in der ersten Hälfte des Jahres 1949 betroffen gewesen und hätten bis zum Jahresende auch nur zum Teil den Wiedereinstieg ins Berufsleben gefunden ${ }^{14}$. Im September 1949 leiteten die Landesverwaltungen eine Kampagne mit dem Ziel ein, den Frauenanteil in der Industrie zu erhöhen. In dem Zusammenhang überprüfte etwa das thüringische Ministerium für Arbeit und Sozialwesen bis zum Februar 1950 insgesamt 1384 Betriebe. Dabei wurden - wie Minister Willi Albrecht gegenüber Ministerpräsident Werner Eggerath mitteilte - 8179 Arbeitsplätze festgestellt, die für Frauen geeignet schienen und offenbar „zum größten Teil“ auch durch Frauen besetzt werden konnten ${ }^{15}$.

11 Ebenda.

12 So beanstandete das Staatssekretariat für Innere Angelegenheiten in einem Bericht Ende 1953, daß die „Umsiedler“ an der „allgemeinen Aufwärtsentwicklung der Bevölkerung nicht in vollem Maße Anteil“ genommen hätten, obwohl sie von Anfang an als „gleichberechtigte Staatsbürger behandelt" worden seien. Erst mit dem Umsiedlergesetz 1950 habe sich diese soziale Ungleichheit geändert. BAB, DQ 2/49, Bl. 140-144, hier Bl. 140, Erläuterungen des Staatssekretariats für Innere Angelegenheiten (Abt. Bevölkerungspolitik) vom 20.10. 1953.

13 BAB, DQ 2/1073, Situationsbericht des Ministeriums für Arbeit und Sozialwesen der Landesregierung Sachsen vom 13. 1. 1950 an das Ministerium für Arbeit und Gesundheitswesen, S. 1.

14 Ebenda.

15 ThHStA, Land Thüringen, Büro des Ministerpräsidenten, Bd. 1683-1684, Bl. 338 f., hier Bl. 338, Minister für Arbeit und Sozialwesen des Landes Thüringen am 25.3. 1950 an den Ministerpräsidenten. 
Um die berufliche Frauenförderung zwischen den Fachministerien besser abstimmen zu können, beabsichtigte das Ministerium für Arbeit und Gesundheitswesen, einen Arbeitsausschuß zu bilden, „der in monatlichen Tagungen die jeweils erforderlichen Aufklärungsmaßnahmen beschließt und die einzelnen Aufgaben verteilt "16. Neben zahlreichen Ministerien ${ }^{17}$ sollten dem Gremium auch die Massenorganisationen (FDGB, DFD, FDJ, Kulturbund, VdgB) angehören. Innerhalb des Ministeriums wurde die Abteilung Mutter und Kind mit der Federführung beauftragt $t^{18}$, die umgehend Vorschläge für ein Arbeitsprogramm des geplanten Ausschusses ausarbeitete ${ }^{19}$. Diese beschränkten sich auf Propagandaaktivitäten in Presse, Rundfunk, Film und Ausstellungen. Der Erfolg dieser Maßnahmen war jedoch sehr stark von der Mitarbeit der übrigen Abteilungen des Arbeitsministeriums, der beteiligten Fachministerien aber auch der Landes- und Kommunalverwaltung abhängig. Die Abteilung Mutter und Kind verfügte nicht über die personellen Ressourcen, um die ambitionierte Zielsetzung alleine durchführen zu können. Das größte Hindernis befand sich aber auf betrieblicher Ebene, da hier über die zusätzliche Beschäftigung von Frauen letztlich entschieden wurde. Gegenüber dem Zentralausschuß für Berufsausbildung bestand von Anfang an eine klare Aufgabentrennung: Während der Zentralausschuß die berufliche Qualifizierung von Frauen vorantreiben sollte, fiel dem Arbeitsausschuß zur Förderung der Frauenarbeit die Aufgabe zu, „die Vorurteile weiter Bevölkerungskreise [...] durch Aufklärung beseitigen [zu] helfen"20. Die Tätigkeit des neu geschaffenen Gremiums beschränkte sich daher auf die Planung und Durchführung von Werbe- und Propagandamaßnahmen. Der Vorschlag der FDJ-Vertreterin Lesser, auf die Bildung des Arbeitsausschusses zu verzichten und statt dessen die Mitarbeit im Zentralausschuß zu verstärken und dessen Aufgabenfeld zu erweitern, fand dagegen keine Unterstützung21.

Auch das Ministerium für Planung erkannte im Sommer 1950, daß der verstärkte Fraueneinsatz in der Wirtschaft nicht reibungslos verlief und die erhofften Erfolge ausblieben. Während die Zahl der vollerwerbsfähigen arbeitsuchenden Männer kontinuierlich gesunken war, hatte bei den erwerbsfähigen Frauen ein gegenläufiger Prozeß eingesetzt ${ }^{22}$. Dem stetigen Ansteigen der Zahl arbeitsuchender Frauen, das eigentümlicherweise nur mit den Folgen der Währungsreform 1948 in kausalen Zusammenhang gebracht wurde, entsprach kein erweitertes Arbeitsplatzangebot. In der Konsequenz stieg die Arbeitslosenquote bei Frauen. Einzelne Landesverwaltungen wie etwa in Sachsen waren daher dazu übergegangen, im Zuge von sogenannten Umsetzungen und durch einen zwischenbezirklichen Ausgleich den beruflichen Einsatz von Frauen zu forcieren, um dadurch den

16 BAB, DQ 2/1073, Vermerk von Minister Steidle vom 25. 5. 1950.

17 Ministerium des Innern, Ministerium für Planung, Ministerium für Industrie, Ministerium für Land- und Forstwirtschaft, Ministerium für Volksbildung, Ministerium für Verkehr, Ministerium für Post- und Fernmeldewesen, Ministerium für Arbeit und Gesundheitswesen, Amt für Information.

18 BAB, DQ 2/1073, Aktenvermerk der Abt. II a vom 6. 6. 1950.

19 Ebenda, Vorschläge der Abt. Mutter und Kind vom 6. 6. 1950.

20 Ebenda, Protokoll über die 1. Sitzung des Arbeitsausschusses zur Förderung der Frauenarbeit, S. 4.

21 Ebenda.

$22 \mathrm{BAB}, \mathrm{DE}$ 1/10105, Bl. 1-7, hier Bl. 2, Bericht des Ministeriums für Planung vom 22. 6. 1950. 
Bedarf an männlichen Arbeitskräften mittelfristig abdecken zu können. Die Arbeitsämter wurden aufgefordert, zunächst einmal regelmäßige Betriebsbegehungen durchzuführen, um den konkreten Arbeitskräftebedarf und die Möglichkeiten zur "Umsetzung" von weiblichen Beschäftigten genau festzustellen. Das Ministerium für Planung mußte jedoch feststellen, daß sich zahlreiche Arbeitsämter an die erteilten Anweisungen nicht hielten und nur vereinzelte Untersuchungen in den Betrieben vornahmen. Dadurch blieb der Erfolg dieser Aktion, die zudem mit einem erheblichen Verwaltungsaufwand verbunden war, von vornherein sehr stark begrenz $t^{23}$. Auch die eingeleiteten Maßnahmen zur Umschulung von weiblichen Arbeitskräften wurden offensichtlich in den Ländern „sehr schlecht" durchgeführt. Auch hierbei erwiesen sich die Betriebe als eigentliches Hindernis: „Die Aufnahme weiblicher Umschüler wird von VEB und SAG unter der Begründung, daß die Senkung der Selbstkosten dadurch gefährdet sei und unter Hinweis auf eine mangelnde Arbeitsdisziplin der Frauen, sehr häufig abgelehnt." 24

Die Abteilung Mutter und Kind, die bekanntlich den Arbeitsausschuß zur Förderung der Frauenarbeit anleitete, begann rasch ein Grundsatz- und Arbeitsprogramm auszuarbeiten. Demzufolge war es erklärtes Ziel, ,im Laufe der nächsten Jahre" etwa 250000 Arbeitskräfte aus dem Kreis der nichterwerbstätigen Frauen zu gewinnen ${ }^{25}$. Nach Einschätzung der Abteilung des Arbeitsministeriums umfaßte der relevante Personenkreis insgesamt rund 2 Millionen erwerbsfähige Frauen, die von der Arbeit freigestellt worden waren, sowie 400000 voll arbeitsfähige Frauen, die bisher keine Beschäftigung gesucht hatten. Die Beschäftigungspraxis der volkseigenen und der privaten Betriebe hatte in der Vergangenheit jedoch mehrfach deutlich werden lassen, daß auch unter dem Einsatz von Werbung und Propaganda nicht zwangsläufig mit einem Erfolg arbeitsmarktpolitischer Programme zu rechnen war. Dieser Umstand wurde von der Abteilung Mutter und Kind aber nicht weiter berücksichtigt ${ }^{26}$, und auch die übrigen $\mathrm{Ab}-$ teilungen des Arbeitsministeriums wiesen auf die problematische Ausgangslage ihrerseits nicht hin. Käthe Kern, die Leiterin der Abteilung Mutter und Kind, präsentierte das Grundsatz- und Arbeitsprogramm auf der zweiten Sitzung des Arbeitsausschusses am 6. Juli 1950. Dabei hob sie besonders hervor, daß vorwiegend Hausfrauen und ehemals berufstätige Frauen als Arbeitskräfte zu gewinnen $\operatorname{seien}^{27}$. Dazu müßten betriebliche Sozialeinrichtungen geschaffen werden, damit die Betreuung von Klein- und Schulkindern gesichert und die Mütter entlastet würden. Kern gelangte zu der überraschenden Einschätzung, die bisher vorliegenden Meldungen der Landesverwaltungen hätten gezeigt, daß Frauen auch in anderen, d.h. berufsfremden Beschäftigungsverhältnissen einsteigen könnten. Dagegen hatten jedoch die Landesministerien in ihren Berichten sowohl das Arbeitsplatzangebot für Frauen als auch die berufliche Mobilität stark in Zweifel gezogen. Letztlich blieb es dabei: Der Arbeitsausschuß beschäftigte sich weniger

3 Ebenda, Bl. $2 \mathrm{f}$.

4 Ebenda, Bl. 4.

25 BAB, DQ 2/1073, Denkschrift der Abt. Mutter und Kind vom 5. 7. 1950, S. 1.

$26 \mathrm{Vgl}$. ebenda, Arbeitsprogramm der Abt. Mutter und Kind für den Arbeitsausschuß vom 5. 7. 1950.

$27 \mathrm{BAB}, \mathrm{DQ} 2 / 1073$, Protokoll über die 2. Sitzung des Arbeitsausschusses am 6. 7. 1950, S. 2. 
mit arbeitsmarktpolitischen Maßnahmen zugunsten der Frauen als vielmehr mit der Propagierung bestehender arbeitsrechtlicher Bestimmungen. Die einzige konkrete Forderung, die auch Käthe Kern wiederholt vorgebracht hatte, bezog sich auf den Ausbau des betriebsgebundenen Kindergartennetzes in der DDR, um Frauen den Einstieg in die Arbeitswelt prinzipiell zu erleichtern. Diese inhaltliche Ausrichtung des Gremiums, an dem sich die Fachministerien offenbar nur noch formell beteiligten, spiegelte sich auch in einem weiteren Arbeitsprogramm wider, das die Vorsitzende am 16. August vorlegte 28 . Darin wurde das Ministerium für Planung allerdings aufgefordert, in Zusammenarbeit mit dem Ministerium für Arbeit und Gesundheitswesen den Frauenanteil in den jeweiligen Arbeitskräfteplänen der volkseigenen Betriebe festzulegen ${ }^{29}$. Diese Aufgabenstellung war auf den ersten Blick neu, entsprach aber durchaus den Zielen der allgemeinen Wirtschaftsplanung. Von entscheidender Bedeutung war, inwieweit es gelang, die aufgestellten Prozentsätze gegenüber den Betrieben durchzusetzen. Das Arbeitsprogramm enthielt die utopische Forderung, den Anteil der weiblichen Beschäftigten in der Verwaltung bis zum Ablauf des Fünfjahrplanes auf mindestens 50 Prozent zu steigern ${ }^{30}$.

In der DDR sank nach Berechnungen des thüringischen Ministeriums für Arbeit und Sozialwesen der Frauenanteil an den Gesamtbeschäftigten zwischen Juni 1949 und Juni 1950 von 37,3 auf 36,4 Prozent. Dabei ergaben sich unterschiedliche Entwicklungen in den einzelnen Ländern ${ }^{31}$ : Während Sachsen einen geringen Rückgang um 0,9 Prozent meldete, lag er in Mecklenburg am höchsten (-3 Prozent); dazwischen befanden sich Sachsen-Anhalt ${ }^{32}$ ( -1 Prozent) und Brandenburg $(-1,4$ Prozent). Die thüringische Landesverwaltung verwies mit Genugtuung darauf, daß Thüringen das einzige Land sei, in dem die Frauenerwerbsquote leicht gestiegen sei - von 36,24 auf 36,77 Prozent. Eine wichtige Voraussetzung für die Erhöhung der Frauenerwerbsquote war ohne Zweifel der Bau von Kindergärten und betrieblichen Kinderkrippen. Zusätzliche Investitionsmittel mußten dafür zur Verfügung gestellt werden. Für den Volkswirtschaftsplan 1951 konnten sich die Staatliche Plankommission und das Ministerium für Gesundheitswesen am 30. März 1951 auf die Verteilung der Finanzmittel in Höhe von 50,1 Millionen DM auf die einzelnen Fachministerien einig werden: Die mit Abstand höchsten

28 Ebenda, Arbeitsprogramm für den Arbeitsausschuß, ausgearbeitet von der Abt. Mutter und Kind (Kern) am 16. 8. 1950.

29 Ebenda, S. 2.

30 Ebenda, S. 3. Nach Berechnungen des Ministeriums für Arbeit und Gesundheitswesen (Abt. Mutter und Kind) befanden sich Ende 1949 im Verwaltungsapparat der DDR-Regierung insgesamt 134 Frauen (vom Referenten aufwärts). Auf Landesebene war die sächsische Landesregierung Spitzenreiter (1182 Frauen), gefolgt von Mecklenburg (239), Brandenburg (217), Thüringen (194) und Sachsen-Anhalt (175). BAB, DQ 2/1073, Hausmitteilung der Abt. Mutter und Kind für Minister Steidle vom 16. 12. 1949.

31 ThHStA, Land Thüringen, Büro des Ministerpräsidenten, Bd. 1692-1699, Bl. 284-300, hier Bl. 285, Bericht des Ministeriums für Arbeit und Sozialwesen Thüringens vom 15. 9. 1950 für den Ministerpräsidenten.

32 Nach Angaben des Ministeriums für Wirtschaft der Landesregierung Sachsen-Anhalt betrug der Frauenanteil in der volkseigenen Wirtschaft Ende 1950 23,2\% und sollte innerhalb eines Jahres auf $30,4 \%$ gesteigert werden. BAB, DQ 2/1755, Bericht des Ministeriums für Wirtschaft (HA Arbeit) in Halle an das Ministerium für Arbeit vom 10. 12. 1951, S. 3. 
Summen (10,5 bzw. 11,5 Millionen DM) waren demnach für die Bereiche Kohle, Maschinenbau und Leichtindustrie vorgesehen ${ }^{33}$.

Die Arbeitsverwaltung begann erst im Laufe des ersten Halbjahrs 1951 ihre Propagandaaktivitäten zur Steigerung der weiblichen Beschäftigtenquote massiv zu verstärken. Dabei machte sich wiederum eine gewisse Planungseuphorie breit:

Tabelle 19: prozentualer Anteil der Frauen an der Gesamtzabl der unselbständig Beschäftigten nach Berufsordnungen und-gruppen ${ }^{34}$

\begin{tabular}{lccc}
\hline & & & \\
& 15.1950 & 30.11 .1950 & 15.6 .1951 \\
\hline Ackerbauer, Tierzüchter, Gartenbauer & 49,3 & 51,0 & 50,2 \\
Forst-, Jagd- und Fischereiberufe & 37,8 & 31,4 & 36,7 \\
bergmännische Berufe & 5,9 & 3,5 & 6,6 \\
Steingewinner und -verarbeiter, Keramiker & 21,5 & 23,4 & 23,0 \\
Glasmacher & 30,3 & 30,1 & 30,3 \\
Bauberufe & 5,9 & 6,6 & 6,8 \\
Maurer & - & 0,3 & 0,9 \\
Metallerzeuger und -verarbeiter & 7,0 & 9,0 & 9,1 \\
Dreher & - & 7,0 & - \\
Schlosser & 1,0 & 2,1 & 2,9 \\
Metalleinbauer & 18,9 & 15,5 & 25,2 \\
Elektriker & 6,9 & 8,4 & 9,2 \\
Chemiewerker & 34,6 & 37,4 & 38,4 \\
Kunststoffverarbeiter & 41,8 & 44,4 & 41,5 \\
Holzverarbeiter und zugehörige Berufe & 10,2 & 10,0 & 10,4 \\
Tischler & 1,0 & 1,4 & 1,7 \\
Papierhersteller und -verarbeiter & 61,8 & 62,0 & 62,9 \\
graphische Berufe & 30,3 & 33,1 & 32,7 \\
Textilhersteller und -verarbeiter & 77,3 & 77,2 & 78,4 \\
Lederhersteller & 34,2 & 35,3 & 36,9 \\
Nahrungs- und Genußmittelhersteller & 47,3 & 47,6 & 50,0 \\
Hilfsberufe der Stofferzeugung & 28,9 & 31,3 & 32,2 \\
Ingenieure und Techniker & 3,2 & 3,8 & 3,1 \\
technische Sonderfachkräfte & 48,0 & 42,1 & 45,5 \\
Maschinisten und zugehörige Berufe & 2,1 & 3,2 & 3,1 \\
kaufmännische Berufe & 45,9 & 48,6 & 49,8 \\
Verkehrs- und Gaststättenberufe & 15,5 & 17,4 & 16,4 \\
hauswirtschaftliche Berufe & 99,9 & 99,8 & 99,8 \\
Reinigungsberufe & 92,0 & 91,2 & 91,6 \\
Gesundheitsdienst und Körperpflegeberufe & 69,6 & 71,1 & 72,1 \\
Volkspflegeberufe & 84,3 & 78,5 & 79,0 \\
Verwaltungs- und Büroberufe & 45,2 & 53,2 & 54,4 \\
Rechts- und Sicherheitsberufe & 16,0 & 21,8 & 22,1 \\
Erziehungs- und Lehrberufe (Seelsorger) & 51,7 & 51,2 & 50,8 \\
Bildungs- und Forschungsberufe & 41,7 & 41,4 & 39,6 \\
künstlerische Berufe & 21,1 & 21,1 & 20,4 \\
\hline
\end{tabular}

Quelle: BAB, DQ 2/3804, Bl. 72, Statistik der HA Arbeit vom 27. 9. 1951.

33 BAB, DQ 2/3804, Bl. 76.

34 Bei den Berufsgruppen wurden die DDR-Bezeichnungen übernommen. 
So entwickelte das Arbeitsministerium im Juni 1951 ambitionierte Richtsätze für die Metallindustrie, die einen Frauenanteil zwischen 5 und 20 Prozent vorsahen ${ }^{35}$. Die Umstrukturierung der Arbeitsämter Mitte 1951 warf die statistische Erhebung der Erwerbsbevölkerung insgesamt gesehen etwas zurück. Neben der bereits mehrfach angesprochenen Eingliederung der Arbeitsämter in die Kommunalverwaltungen ist außerdem noch auf die Überleitung des Fachbereichs Nachwuchslenkung in die Zuständigkeit des Staatssekretariats für Berufsausbildung zu verweisen. So verwunderte es auch nicht, als Abteilungsleiter Heisig auf Anfrage des Dresdener Lehrstuhls für Betriebswissenschaften und Normung betonte, daß eine Aufstellung der gelernten, angelernten sowie der fachlich qualifizierten weiblichen Arbeitskräfte momentan nicht möglich sei ${ }^{36}$. Statt dessen lieferte er eine Statistik, die den prozentualen Frauenanteil in den einzelnen Berufsgruppen enthielt und somit zumindest die Schwerpunkte der Frauenbeschäftigung deutlich werden ließ.

Nach Auswertung der betrieblichen Personalkarteien kam die Abteilung Arbeitskraftlenkung zum Ergebnis, daß der Anteil der beschäftigten Frauen in der volkseigenen Wirtschaft Ende 1951 durchschnittlich 30,4 Prozent betrug37. Der im Volkswirtschaftsplan 1951 festgesetzte Frauenanteil von 32 Prozent war somit nicht erreicht worden. Vor allem in folgenden Wirtschaftsbereichen konnte das Planziel mit Abstand nicht erfüllt werden: Elektrotechnik, Feinmechanik und Optik, Chemie, Bauindustrie, Holzverarbeitung, Textilindustrie, polygraphische Industrie und Lebensmittelindustrie. Als Gründe für diese Entwicklung gab die Fachabteilung des Arbeitsministeriums als erstes an, daß zahlreiche Betriebe es nach wie vor ablehnen würden, Frauen in Berufen, die zuvor Männern vorbehalten waren, zu beschäftigen. Gleichzeitig wurde die fehlende berufliche Qualifizierung betont, die eine Anstellung in Facharbeiterberufen von vornherein ausgeschlossen hätte. Negative Auswirkungen für die Frauenbeschäftigtenrate hatten offensichtlich auch die zunehmende Entwicklung der Arbeitsproduktivität, die mit einer Rationalisierung und einem Stellenabbau teilweise einherging, sowie Veränderungen der Produktionsauflagen, die zu einer Veränderung der Arbeitskräftepläne geführt hatten. Abschließend verwies die Abteilung Arbeitskraftlenkung auf die "noch immer unzureichenden sanitären, sozialen und hygienischen Einrichtungen in den Betrieben, die die Beschäftigung von Frauen erschweren“38. Zuwachsraten beim Frauenanteil konnten in der Privatwirtschaft ${ }^{39}$ erzielt werden, während die Quote in der Landwirtschaft gesunken war ${ }^{40}$, sich aber immer noch auf relativ hohem Niveau befand.

Nachdem der Bericht auch dem DDR-Ministerrat vorgelegt worden war, beschäftigte sich dieser Anfang Mai 1952 mit arbeitsmarktpolitischen Maßnahmen zur Steigerung der Frauenerwerbsquote und faßte einen Beschluß „über die Ein-

$35 \mathrm{BAB}, \mathrm{DQ} 2 / 1859$.

$36 \mathrm{BAB}, \mathrm{DQ} 2 / 3804, \mathrm{Bl} .70 \mathrm{f}$., Abteilungsleiter Heisig am 1. 10. 1951 an den Lehrstuhl für Betriebswissenschaften und Normung der TH Dresden (Dipl.-Ing. Weinhold).

$37 \mathrm{BAB}, \mathrm{DQ} 2 / 844$, Bericht der Abt. Arbeitskraftlenkung vom 28. 4. 1952 an die SPK (Planung der Arbeitskräfte), S. 1 .

38 Ebenda.

39 Ebenda, S. 2. Bei den SAG-Betrieben war der Frauenanteil von 22 auf $21,8 \%$ zurückgegangen.

tc Von 55,1\% (Dez. 1950) auf 47,5\% (Dez. 1951). Ebenda, S. 3. 
beziehung von Frauen in den Arbeitsprozeß ${ }^{41}$. Darin wurde das Ministerium für Arbeit beauftragt, dafür zu sorgen, daß die Abteilungen für Arbeit bei den Räten der Stadt- und Landkreise die betrieblichen Kontrollen wieder verstärkt aufnehmen, damit der festgelegte Frauenanteil auch eingehalten wird ${ }^{42}$. Sollten die Kontrollen ergeben, daß die Betriebe durchaus in der Lage sind, über die im Arbeitskräfteplan enthaltenen Quoten hinaus mehr Frauen einzustellen, waren die Betriebsleitungen dazu zu veranlassen. Darüber hinaus sollten in allen Betrieben „Arbeitsplatzanalysen“ durchgeführt werden, um die Arbeitsplätze zu ermitteln, die besonders für Frauen geeignet waren. Die Fachministerien und Staatssekretariate wurden angehalten, dem Ministerium bei der Durchführung dieser Maßnahmen behilflich zu sein ${ }^{43}$. Zwischen dem 31. Mai und dem 30. August 1952 führte das Ministerium für Arbeit nach eigenen Angaben insgesamt 2799 Betriebsüberprüfungen durch (1524 VEB und 1275 Privatbetriebe) ${ }^{44}$. Auch wenn die Entwicklung im ersten Halbjahr 1952 hinter den gesteckten Planzielen insgesamt zurückblieb, konnte doch zumindest in einzelnen Wirtschaftsbranchen ein aus Sicht der Planungsverwaltung befriedigendes Zwischenergebnis vorgelegt werden: Letzteres galt unter anderem für die Plangruppen Bergbau, Guß- und Schmiedehandwerk, Grundchemie, Leder- und Bekleidungsindustrie sowie Bauindustrie45. Im dritten Quartal 1952 lagen weitere punktuelle Verbesserungen vor: So war es offenbar gelungen, das Planziel in der volkseigenen örtlichen Industrie fast zu erreichen (32,1 gegenüber 32,9 Prozent) ${ }^{46}$. Dagegen war das Planziel in der volkseigenen zentral gelenkten Industrie noch nicht erreicht worden (32,6 gegenüber 36,7 Prozent). Für die SAG-Betriebe waren die Vorgaben nach unten korrigiert worden (20,0 Prozent), so daß hier ebenfalls ein positives Ergebnis (23,4 Prozent) erzielt werden konnte. Bei diesen Angaben darf allerdings nicht übersehen werden, daß etwa in der volkseigenen zentral gelenkten Industrie die Beschäftigtenzahl insgesamt angestiegen war ${ }^{47}$. Problematisch war immer noch die berufliche Aus- und Weiterbildung von Frauen: Das Ministerium für Arbeit stellte dazu fest, daß die „große Masse der Frauen Ungelernte bzw. Hilfsarbeiterinnen ohne jede Qualifikation" seien ${ }^{48}$. Die überwiegende Mehrzahl der weiblichen Beschäftigten befand sich in den unteren Lohngruppen.

4 BAB, DC 20 I/3-105, Bl. 4, Protokoll der Sitzung der Regierung der DDR vom 2. 5. 1952.

42 Ebenda, Bl. 79f., Beschluß zum Bericht über Maßnahmen zur Einbeziehung von Frauen in den Produktionsprozeß, vom Ministerium für Arbeit am 26. 4. 1952 vorgelegt.

43 In der Folgezeit gab es vereinzelt Arbeitstagungen, zu denen das Arbeitsministerium die Leiter der Abteilungen Arbeit der Fachministerien und Staatssekretariate einlud. Dabei sprachen sich Vertreter der Fachministerien für eine Lockerung der Arbeitsschutzbestimmungen aus: So dürften Frauen im EKO nicht am Hochofen arbeiten, obwohl sie fachlich qualifiziert seien. Im Mansfelder Kupferbergbau dürften Frauen nicht untertage arbeiten, „obwohl es dort eine Anzahl leichte Arbciten zu verrichten gibt und sich auch viele Frauen dazu bereit erklärt haben." BAB, DQ 2/1866, Protokoll über die Arbeitstagung vom 9. 10. 1952. Die Leiterin der Abt. Mutter und Kind, Käthe Kern, hatte sich frühzeitig für Ausnahmeregelungen beim bestehenden Verbot von Nachtarbeit für Frauen ausgesprochen. BAB, DQ 2/1073, Vermerk Kerns vom 18. 11. 1949.

44 BAB, DQ 2/844, Minister Chwalek am 4. 10. 1952 an Ministerpräsident Grotewohl, S. 1.

45 Ebenda, S. $5 \mathrm{f}$.

$46 \mathrm{BAB}, \mathrm{DQ} 2 / 844$, Bericht des Ministeriums für Arbeit vom 15. 1. 1953, S. 2.

47 Ebenda.

48 Ebenda, S. 6. 
Tabelle 20: Frauenanteil (in Prozent) in einzelnen Lohngruppen (III. Quartal 1952)

Lohngruppe I Lohngruppe II Lohngruppe VII Lohngruppe VIII

\begin{tabular}{lcccc}
\hline HV Allgemeiner & 81,6 & 80,1 & 0,6 & 0,5 \\
Maschinenbau & & 77,4 & 0,5 & 1,0 \\
HV Steine und Erden & 75,8 & 87,9 & 0,6 & 0,8 \\
HV Feinmechanik und & 85,0 & & & \\
Optik & & 89,9 & 2,0 & 1,4 \\
HV Elektrotechnik & 91,3 & 91,6 & 1,7 & 3,6 \\
HV Chemie & 91,9 & 28,3 & 0,2 & 0,2 \\
HV Bauindustrie & 30,8 & &
\end{tabular}

Quelle: BAB, DQ 2/844, Bericht des Ministeriums für Arbeit vom 15. 1. 1953, S. 7.

Die Zuversicht der DDR-Regierung, den Anteil der Frauen an der Gesamtbeschäftigtenzahl langfristig und kontinuierlich steigern zu können, wich in zunehmendem Maße einer pragmatischen Politik. So nahm der Ministerrat auf seiner Sitzung am 23. März 1953 Abstand von der bisherigen Quotenfestsetzung in den jeweiligen Volkswirtschaftsplänen. Die Forderung, einen Frauenanteil von durchschnittlich 37 Prozent zu erreichen, wurde fallengelassen, „wenn für die, durch die Erfüllung des planmäßigen Frauenanteils freiwerdenden männlichen Arbeitskräfte anderweitige Arbeitsmöglichkeiten nicht vorhanden sind"49. Voraussetzung für die „Umsetzung“ von männlichen Arbeitskräften war somit das Vorhandensein von neuen Beschäftigungsmöglichkeiten, vor allem in der Schwerindustrie.

\section{Jugendliche}

Die Bereitstellung von Lehrstellen blieb auch nach der DDR-Gründung eine vordringliche Aufgabe der Arbeitsverwaltung, bei der sich ebenfalls die grundsätzlichen Schwierigkeiten einer bedarfsgerechten Steuerung bemerkbar machten. Einzelne Arbeitsämter hatten sich hierbei hilfesuchend an die HVAS bzw. das Ministerium für Arbeit und Gesundheitswesen in Berlin gewandt und waren dabei auch von bürgerlichen Blockparteien unterstützt worden ${ }^{50}$. Auch wenn der Nachwuchsplan 1949 das Ziel verfolgt hatte, die Ausbildung in sämtlichen Berufen zu regeln, traf dies de facto nur für die sogenannten Mangelberufe $\mathrm{zu}^{51}$. Angesichts der Dringlichkeit, die einzelne Wirtschaftsbranchen auch in diesem arbeitsmarktpolitischen Bereich besaßen, kam das Arbeitsministerium letztlich nicht umhin, Prioritäten bei der Unterbringung erwerbsloser Jugendlicher in Lehr- und Arbeitsstellen zu setzen. Eine gleichmäßige Verteilung der jugendlichen Arbeitslosen auf die Kombinate und Betriebe war nicht durchführbar.

$49 \mathrm{BAB}, \mathrm{DQ} 2 / 1760$, Entwurf der Abt. Arbeitskraftlenkung vom 14. 4. 1953, S. 9.

50 So hatte beispielsweise die LDP in Görlitz den Aufbau einer Leichtindustrie in der Stadt bzw. der näheren Umgebung gefordert. Vgl. BAB, DQ 2/906, Bl. 53-58, hier Bl. 55 f., Schreiben des Vorsitzenden des LDP-Stadtverbandes Görlitz (Sommer) vom 21.4. 1949 an den LDP-Vorsitzenden Kastner.

51 BAB, DQ 2/906, Bl.50f., Aktenvermerk des Ministeriums für Arbeit und Gesundheitswesen (Abt. Planung und Statistik) vom 13. 2. 1950. 
Bei der Durchführung des Nachwuchsplanes 1950 gelang es den Arbeitsämtern nur in unzureichendem Maße, Lehrstellenangebot und -nachfrage in Übereinstimmung zu bringen. So wies Ministerpräsident Grotewohl, der vermutlich im September 1950 über diese Fehlentwicklung unterrichtet worden war, darauf hin, daß für Jugendliche unter 18 Jahren ein „ungedecktes Stellenangebot für Sofortbedarf" vorliege, während andererseits noch rund 70000 Jugendliche auf die $\mathrm{Zu}$ weisung eines Arbeitsplatzes warteten ${ }^{52}$. Grotewohl sprach sich dafür aus, die beschäftigungslosen Jugendlichen "durch Austausch“ innerhalb der DDR in Arbeit zu bringen. Nach Angaben des Ministeriums für Arbeit und Gesundheitswesen hatte sich das Arbeitsplatzangebot für Jugendliche im Laufe des Spätsommers 1950 dramatisch verschlechtert: Innerhalb eines Monats war die Zahl der arbeitsuchenden Jugendlichen von 69625 (31. August) auf 92942 (30. September) angestiegen, während die Zahl der offenen Stellen von 13771 auf 3351 gesunken war ${ }^{53}$. Von dieser Entwicklung wurde zwar das Lehrstellenangebot zunächst noch nicht tangiert. Es mußte jedoch damit gerechnet werden, daß zahlreiche Jugendliche, die keine Beschäftigung gefunden hatten, nunmehr eine Lehrstelle suchen würden. Um diesen Negativtrend zu stoppen, schlug Minister Steidle einen Maßnahmenkatalog vor, der jedoch insgesamt gesehen sehr vage blieb. So strebte die Arbeitsverwaltung "weitere Bemühungen [...] zur optimalen Besetzung der gegenwärtig noch vorhandenen freien Arbeitsplätze“ an ${ }^{54}$. Darüber hinaus sollte auf "geeignete Betriebe“ eingewirkt werden, ihr Lehrstellenangebot zu erweitern. Außerdem erwog Steidle eine Jugendlichenquote einzuführen: Ähnlich wie bei den Schwerbeschädigten sollten die Betriebe gezwungen werden, eine bestimmte Anzahl von Jugendlichen einzustellen. Als letztes war die öffentliche Propagierung der überbezirklichen Lenkung beabsichtigt. Mit Hilfe von „Werbung und Aufklärung" sollte unter den Jugendlichen und Eltern die Bereitschaft zum Wohnortwechsel geweckt bzw. gefördert werden. Ministerpräsident Grotewohl gab seine Zustimmung zu den geplanten Maßnahmen der Arbeitsverwaltung und plädierte dafür, die mit dem Nachwuchsplan zusammenhängenden Fragen zusammen mit den Blockparteien und Massenorganisationen zu erörtern: „Die Erfahrungen im Erzbergbau zeigen uns, daß dieses Verfahren allein richtig ist, um Probleme, wie sie jetzt wieder bei der Lehrlingsausbildung vor uns stehen, einer Lösung zuzuführen, die nur dann gut ist, wenn sie vom Verständnis der gesamten Bevölkerung getragen wird. “ ${ }^{5}$ Nach Ansicht Grotewohls sollten daher „im Block der antifaschistisch-demokratischen Parteien und Organisationen unter besonderer Heranziehung " der FDJ die "notwendigen Entscheidungen" herbeigeführt werden. In der Folgezeit wurde dieser Weg zur Mobilisierung der Bevölkerung genutzt, die inhaltlichen Beschlüsse trafen allerdings nach wie vor die Arbeitsverwaltung sowie die Staatliche Plankommission.

52 SAPMO, NY 4090/561, Bl. 118, Grotewohl am 6. 10. 1950 an Minister Steidle.

53 Ebenda, Bl. 119-121, hier Bl. 120, Ministerium für Arbeit und Gesundheitswesen am 26. 10. 1950 an Ministerpräsident Grotewohl.

54 Ebenda.

55 SAPMO, NY 4090/561, Bl. 122, Grotewohl am 31. 10. 1950 an Minister Steidle. 
Tabelle 21 a: Übersicht über die Beschäftigungslage unter Jugendlichen (Stand: 31. Oktober 1950)

\begin{tabular}{|c|c|c|c|c|c|}
\hline & & männ & & & \\
\hline & $\begin{array}{l}\text { ugendliche Art } \\
\text { rollarbeitsfähig }\end{array}$ & $\begin{array}{l}\text { eitslose } \\
\text { erwerbsbeschr. }\end{array}$ & zusammen & $\begin{array}{l}\text { offene Stellen } \\
\text { Lehrstellen }^{56}\end{array}$ & Arbeitsplätze \\
\hline Sachsen & 4301 & 1223 & 5524 & 4991 & 350 \\
\hline Sachsen-Anhalt & 9034 & 830 & 9864 & 2860 & 600 \\
\hline Thüringen & 3832 & 437 & 4269 & 1851 & 257 \\
\hline Brandenburg & 6580 & 22 & 6602 & 785 & 214 \\
\hline Mecklenburg & 2738 & 151 & 2889 & 540 & 598 \\
\hline DDR & 26485 & 2663 & 29148 & 11027 & 2019 \\
\hline
\end{tabular}

Quelle: SAPMO, NY 4090/561, Bl. 124, Übersicht des Ministeriums für Arbeit und Gesundheitswesen (Abt. Planung und Statistik).

Tabelle 21 b: Übersicht über die Beschäftigungslage unter Jugendlichen (Stand: 31. Oktober 1950)

\begin{tabular}{|c|c|c|c|c|c|}
\hline & & weibl & & & \\
\hline & $\begin{array}{l}\text { ugendliche Arl } \\
\text { ollarbeitsfähig }\end{array}$ & $\begin{array}{l}\text { eitslose } \\
\text { erwerbsbeschr. }\end{array}$ & zusammen & $\begin{array}{l}\text { offene Stellen } \\
\text { Lehrstellen } 57\end{array}$ & Arbeitsplätze \\
\hline Sachsen & 11038 & 1377 & 12415 & 2331 & 150 \\
\hline Sachsen-Anhalt & 20822 & 843 & 21665 & 805 & 440 \\
\hline Thüringen & 6860 & 352 & 7212 & 998 & 262 \\
\hline Brandenburg & 11455 & 57 & 11512 & 124 & 124 \\
\hline Mecklenburg & 3822 & 228 & 4050 & 80 & 146 \\
\hline DDR & 53997 & 2857 & 56854 & 4338 & 1122 \\
\hline
\end{tabular}

Quelle: SAPMO, NY 4090/561, Bl. 124, Übersicht des Ministeriums für Arbeit und Gesundheitswesen (Abt. Planung und Statistik).

Als problematisch erwies sich nicht nur die Versorgung der Jugendlichen mit Lehrstellen, sondern auch die berufliche Unterbringung der Jugendlichen, die ihre Lehre abgeschlossen hatten. Zahlreiche Betriebe sahen sich nicht in der Lage, eine Übernahmegarantie auszusprechen. Nachdem das Ministerium für Arbeit auf die drohende Erwerbslosigkeit der ehemaligen Lehrlinge aufmerksam gemacht worden war, trafen sich Anfang April 1951 Vertreter der an der Berufsausbildung mitwirkenden Ministerien bzw. Staatssekretariate sowie des FDGB und der FDJ, um Gegenmaßnahmen zu entwickeln. Es müsse - so der Mitarbeiter des Ministeriums für Arbeit Hecker - oberstes Ziel sein, ein Ansteigen der Jugendarbeitslosigkeit zu verhindern ${ }^{58}$. Des weiteren dürften Frauen, die "mit viel Mühe in einen Männerberuf" gelangt seien, ihren Arbeitsplatz nicht wieder verlieren. Konkrete Beschlüsse wurden jedoch auf dieser Sitzung nicht gefaßt; statt dessen erhielten die

56 Angegeben sind geschätzte Zahlengrößen.

57 Angegeben sind geschätzte Zahlengrößen.

$58 \mathrm{BAB}, \mathrm{DQ} 2 / 1848$, Protokoll über die Sitzung am 3. 4. 1951, S. 2. 
Fachministerien den unverbindlichen Auftrag, den Arbeitskräftebedarf in den Betrieben festzustellen ${ }^{59}$. Die DDR-Regierung hatte bekanntlich das Ministerium für Arbeit am 19. April ermächtigt, die Entlassung von Lehrlingen zu verbieten. In Ausführung dieses Beschlusses versandte Minister Chwalek zwei Monate später eine Rundverfügung, die eine umfassende Arbeitsplatzgarantie für die Jugendlichen enthielt ${ }^{60}$. So war unter anderem auch die Arbeitsplatzzuweisung in einem anderen Betrieb geplant, falls der auszubildende Betrieb den Lehrling nicht weiter beschäftigen konnte. Um eine Verringerung der Lehrstellen zu unterbinden, wies Chwalek die Verwaltungen an, daß die Weiterbeschäftigung von jungen Facharbeitern nicht zu einer Reduzierung der Ausbildungsplätze führen dürfte. Die Rundverfügung enthielt allerdings keine Fristenregelungen und sah auch keine Sanktionsmaßnahmen gegenüber Betrieben vor, die dieser Anweisung nicht Folge leisteten.

Während es der Arbeitsverwaltung 1951 halbwegs gelungen war, eine größere Entlassungswelle zu verhindern, zeichnete sich im Frühjahr des folgenden Jahres ein Scheitern der bisherigen arbeitsmarktpolitischen Maßnahmen auf diesem Gebiet ab. Die noch bestehenden Landesregierungen lieferten dem Berliner Arbeitsministerium zum Teil alarmierende Berichte über die gesunkenen Chancen junger Facharbeiter auf den Teilarbeitsmärkten der DDR. Davon waren auch größere Werke bzw. Kombinate der Schwerindustrie betroffen, wie etwa das Stahlwerk Riesa oder das Braunkohlenwerk Böhlen ${ }^{61}$. Die Werksleitungen sahen sich offenbar außerstande, die Lehrlinge nach Abschluß der Ausbildung als Facharbeiter zu übernehmen. Damit war auch die anfängliche Euphorie, den zukünftigen Facharbeiterbedarf mittelfristig zu planen, einer Ernüchterung gewichen. Die Betriebe, die ungefähr zeitgleich zur weiteren Steigerung der Arbeitsproduktivität angehalten worden waren, konnten sich mit einer erheblichen Vergrößerung des Facharbeiterbestandes nicht einverstanden erklären. $\mathrm{Da}$ die Auftragslage von den $\mathrm{Be}-$ triebsleitungen im Frühjahr 1952 pessimistisch eingeschätzt wurde, sah die Arbeitsverwaltung in der Ausweitung der Produktionsauflagen den einzigen Ausweg. So rechnete das sächsische Ministerium mit einer Weiterbeschäftigung aller Lehrlinge im Stahlwerk Riesa, falls es gelänge, die Inbetriebnahme des neu errichteten Rohrwerkes zeitlich vorzuziehen ${ }^{62}$. Die frühzeitige Entlassung von älteren Facharbeitern, die kurz vor der Verrentung standen, wurde dagegen nie ernsthaft erwogen: Sowohl die Arbeitsverwaltung als auch die Betriebe sprachen sich gegen eine solche Maßnahme aus ${ }^{63}$.

Im Sommer 1952 beabsichtigte das Ministerium für Arbeit, eine weitere Rundverfügung herauszugeben, die sich inhaltlich sehr stark an der ersten Fassung vom 20. Juni 1951 orientieren sollte ${ }^{64}$. Darüber hinaus wurde im Entwurf den Betrie-

59 Ebenda, S. 10.

$60 \mathrm{BAB}, \mathrm{DQ} 2 / 1848$, Rundverfügung über die Weiterbeschäftigung von jungen Facharbeitern nach Abschluß ihrer Ausbildungszeit vom 20.6.1951.

61 Ebenda, Ministerium für Wirtschaft und Arbeit des Landes Sachsen am 7.3. 1952 an Minister Chwalek.

62 Ebenda, S. 1.

63 $\mathrm{BAB}, \mathrm{DQ} 2 / 1849$, Ministerium für Wirtschaft und Arbeit des Landes Sachsen am 2. 5. 1952 an Minister Chwalek, S. 1.

$64 \mathrm{BAB}, \mathrm{DQ} 2 / 1848$, Entwurf einer Rundverfügung der Abt. Arbeitskraftlenkung vom 3. 7. 1952. 
ben das Recht eingeräumt, junge Facharbeiter berufsfremd einsetzen zu können. Durch innerbetriebliche Selbstregulierung sollte somit die Weiterbeschäftigung gewährleistet bleiben. Eine vertrauliche Anordnung vom 10. Juli sah zunächst die Möglichkeit der Entlassung älterer Arbeiter vor; die Kündigung durfte jedoch erst beim Nachweis eines ,ihren Kenntnissen und Fähigkeiten entsprechenden Arbeitsplatzes" erfolgen ${ }^{65}$. Ende August ließ sich der Ministerrat von Staatssekretärin Malter über „die Einbeziehung der vom Plan der Berufsausbildung nicht erfaßten Jugendlichen in den Arbeitsprozeß" unterrichten und faßte einen Beschluß, in dem die beteiligten Ressorts aufgefordert wurden, die Ausbildung in den Berufsschulen zu verbessern ${ }^{66}$. Darüber hinaus wurden das Arbeitsministerium und die Fachministerien - wie bereits erwähnt - damit beauftragt, die vor der Entlassung stehenden Lehrlinge statistisch $\mathrm{zu}$ erfassen und weitere Qualifizierungslehrgänge für diese Jugendlichen durchzuführen ${ }^{67}$. Der DDR-Ministerrat vermied es aber, konkrete Vorgaben für die Übernahme von ehemaligen Lehrlingen durch die Betriebe aufzustellen. Das grundsätzliche Problem der weiteren Beschäftigung von Jugendlichen nach Abschluß der Lehre wurde offensichtlich nicht einmal thematisiert.

Die Chancen zur Unterbringung von auslernenden Lehrlingen sahen in den einzelnen Wirtschaftsbranchen unterschiedlich aus und hingen vom vorgegebenen Arbeitskräfteplan sowie der Auftragslage ab. Zahlreiche Betriebe des Industriezweiges Kohle und Energie sperrten sich gegen eine Beschäftigung von Lehrlingen, „die über den Bedarf vorhanden sind“, befürchteten sie doch einen Rückgang der Arbeitsproduktivität infolge der Einarbeitungsphase, die den Berufsanfängern gewährt werden mußte ${ }^{68}$. Am betrieblichen Widerstand scheiterte letztlich oftmals die von den Ministerien eingeleitete sogenannte Umsetzung der ausgebildeten Facharbeiter. Das Ministerium für Arbeit vermied es jedoch, eine grundsätzliche Anweisung zu erlassen, und verabschiedete statt dessen Richtlinien „über die Qualifizierung der nicht vom Plan der Berufsausbildung erfaßten Jugendlichen in

65 Ebenda, Anordnung der Abt. Arbeitskraftlenkung vom 10.7. 1952, S. 1 ( $\$ 1$, Abs. 2).

$66 \mathrm{BAB}, \mathrm{DC} 20 \mathrm{I} / 3-128, \mathrm{Bl} .7$ und 33f., Protokoll der Sitzung der Regierung der DDR am 28. 8. 1952.

67 Ebenda, Bl. $36 f$.

68 Das EKO sah sich nur unter der Bedingung zur Übernahme weiterer Jungfacharbeiter aus anderen Betrieben bereit, daß für jeden ausgebildeten Lehrling ein erfahrener Facharbeiter zur Verfügung gestellt würde. BAB, DQ 2/1839, Staatssekretariat für Kohle und Energie (Hauptreferat Schulung) am 9. 9. 1952 an das Ministerium für Arbeit. Diese Forderung hätte jedoch eine gründliche Überarbeitung der Arbeitskräftepläne nach sich gezogen und war daher unrealistisch. Darüber hinaus wandten sich auch einzelne Walzwerke an das zuständige Fachministerium, um mitzuteilen, daß die Weiterbeschäftigung der noch in Ausbildung stehenden Jugendlichen nicht möglich sei. Die Ministerien wurden in solchen Fällen gebeten, Maßnahmen zu ergreifen, um diese Lehrlinge in andere Betriebe umzusetzen. Vgl. BAB, DQ 2/1839, VEB Metallschmelz- und Walzwerk Merseburg am 20.11. 1952 an das Ministerium für Hüttenwesen und Erzbergbau (Abt. Arbeit); ebenda, Stahl- und Walzwerk Riesa am 24.11. 1952 an das Ministerium für Hüttenwesen und Erzbergbau (Abt. Arbeit). Aus Sicht des Ministeriums für Arbeit erhöhte sich der Handlungsdruck noch dadurch, daß nicht nur die Fachministerien die betrieblichen Meldungen weiterreichten, sondern auch die Bezirksverwaltungen entsprechende Anfragen nach Berlin richteten. So meldete der Rat des Bezirkes Gera einen Überhang von ausgebildeten Facharbeitern bei der Maxhütte in Unterwellenborn: Dort konnten von 80 Walzlehrlingen, die im November 1952 ihre Facharbeiterprüfung ablegten, nur fünf übernommen werden. Ein ähnlicher Vorgang wurde für das nachfolgende Lehrjahr prognostiziert: Von insgesamt 180 Lehrlingen sollten nur vier weiterhin im Werk beschäftigt werden. Vgl. BAB, DQ 2/1839, Rat des Bezirkes Gera am 6.12. 1952 an Minister Chwalek. 
der volkseigenen Wirtschaft", die sich explizit auf den Beschluß des Ministerrates vom 28. August beriefen ${ }^{69}$. Erneut wurden die Werksdirektoren aufgefordert, zunächst einmal die Arbeitsplätze innerhalb des Betriebes zu ermitteln, an denen Jugendliche "unter Berücksichtigung ihrer geistigen und körperlichen Entwicklung, vor allem weibliche Jugendliche“, beschäftigt werden konnten ${ }^{70}$. Die Jugendlichen, denen im Rahmen des jährlichen Nachwuchsplanes kein Ausbildungsplatz zur Verfügung stand, sollten auf die volkseigenen und ihnen gleichgestellten $\mathrm{Be}-$ triebe aufgeteilt und dort angelernt werden. Durch die Schulung müsse erreicht werden, daß die Jugendlichen „eine ordnungsgemäße Ausbildung für Tätigkeiten erhalten, die auf Grund ihrer Kompliziertheit in der Regel den Lohngruppen 2 und 3 angehören "71. Das waren letztlich die unteren Lohngruppen, die aufgrund des Ausbildungsgrades (Angelernte) vorgesehen waren. Minister Chwalek forderte die Industrieministerien auf, auf dieser Grundlage eigene Richtlinien für den jeweiligen Geschäftsbereich auszuarbeiten, welche die Besonderheiten der Wirtschaftszweige zu berücksichtigen hatten ${ }^{72}$.

Nachdem das Ministerium für Arbeit erkannt hatte, daß gegen die Betriebsleitungen eine Ausdehnung des Beschäftigtenbestandes und damit eine Weiterbeschäftigung der Jungfacharbeiter nicht zu erreichen war, sprach sich die innerhalb des Ministeriums zuständige Abteilung Arbeitskraftlenkung dafür aus, die berufliche Qualifizierung noch stärker als bisher von den allgemeinen Berufsschulen in die Betriebe zu verlagern ${ }^{73}$. Dies schien die zwangsläufige Konsequenz aus den zurückliegenden Erfahrungen zu sein, welche das Arbeitsministerium bei seinen Bemühungen zur Unterbindung der Jugendarbeitslosigkeit hatte sammeln müssen. Für einen solchen Schritt sprachen vor allem zwei Gründe: Zum einen konnte der Bedarf an Facharbeitern auf betrieblicher Ebene sehr viel genauer kalkuliert und entsprechend die Zahl der Auszubildenden angepaßt werden. Zum anderen war die Ausbildung in den Betrieben sehr viel praxisbezogener als in den Berufsschulen. Gleichzeitig sah sich allerdings das Ministerium für Arbeit gezwungen, in Absprache mit den zuständigen Fachministerien kurzfristige und pragmatische Lösungen für die betroffenen Kombinate und Betriebe zu finden. So konnte nach mehrmaliger Rücksprache mit dem Ministerium für Hüttenwesen und Erzbergbau erreicht werden, daß die ausgebildeten Facharbeiter der Maxhütte (Unterwellenborn), welche dort nicht übernommen werden konnten, in verschiedenen Blechwalzwerken innerhalb der DDR zunächst zum Blechwalzwerker umgeschult und anschließend im EKO eingestellt wurden ${ }^{74}$.

Mit einer grundsätzlichen Verbesserung der Beschäftigungslage für ehemalige Lehrlinge rechnete das Berliner Arbeitsministerium insgesamt jedoch nicht. Im Gegenteil: Die Situation würde sich - so die pessimistische Einschätzung - Anfang 1953 weiter verschlechtern. Die Abteilung Arbeitskraftlenkung ging davon

$69 \mathrm{BAB}, \mathrm{DQ} 2 / 1839$, Richtlinien des Ministeriums für Arbeit vom 30. 9. 1952.

70 Ebenda, S. 2.

71 Ebenda, S. 3.

72 BAB, DQ 2/1848, Rundschreiben Chwaleks vom 1. 10.1952.

73 BAB, DQ 2/1852, Abt. Arbeitskraftlenkung am 12. 12. 1952 an den Rat des Bezirkes Magdeburg (Abt. Arbeit und Berufsausbildung).

74 BAB, DQ 2/1839, Abt. Arbeitskraftlenkung am 18.12. 1952 an den Rat des Bezirks Gera (Abt. Arbeit und Berufsausbildung). 
aus, daß von den rund 600 Jugendlichen, die dann ihre Lehrzeit in der Maxhütte sowie zwei weiteren Werken beendet haben würden, "nur ein ganz geringer Teil“ weiterhin in den Werken beschäftigt werden könne ${ }^{75}$. Da die Arbeitsämter durch die Verordnung vom 12. Juli 1951 in ihrer ursprünglichen Form aufgelöst worden waren, warf die IHK Sachsen-Anhalt die Frage auf, ob die Verteilung der Arbeitskräfte nicht grundsätzlich neu zu regeln sei, und zwar mit einer entsprechenden Stärkung der VVB bzw. VEB ${ }^{76}$. Auf diese Weise hätte sich das Arbeitsministerium nahezu vollständig aus der bisherigen Aufgabe, die berufliche Unterbringung von Jugendlichen zu gewährleisten, verabschiedet. Diesem Vorstoß widersprach das Ministerium für Arbeit vehement und stellte die Ausarbeitung einer weiteren Richtlinie für das Jahr 1953 in Aussicht ${ }^{77}$. In dem Zusammenhang betonte Abteilungsleiter Arndt, daß die Aufgaben, die zuvor die Arbeitsämter wahrgenommen hätten, nunmehr auf die neu gebildeten Abteilungen für Arbeit und Berufsausbildung übergegangen seien. So müßten die Betriebe den in die Kommunalverwaltung eingegliederten Abteilungen drei Monate vor Beendigung der Lehrzeit Mitteilung von der drohenden Entlassung machen. Die Abteilungen für Arbeit und Berufsausbildung würden „dann endgültig über die weitere Unterbringung dieser Jungfacharbeiter" entscheiden. Diese Position spiegelte sich dann auch in einer Beschlußvorlage wider, die Arbeitsminister Chwalek am 15. Januar 1953 dem Chef der Regierungskanzlei unterbreitete ${ }^{78}$. Diese sah eine Beauflagung der Betriebe zur Einstellung von Schwerbeschädigten, Frauen und Jugendlichen vor. Darüber hinaus sollte eine Meldepflicht für die Jugendlichen eingeführt werden, die vom Berufsausbildungsplan nicht erfaßt wurden ${ }^{79}$. Als Begründung für den Konfrontationskurs gegenüber den Betrieben gab das Arbeitsministerium an: „Die Betriebe weigerten sich oftmals, die Bemühungen der Abteilungen für Arbeit und Berufsausbildung zur Einbeziehung von Frauen, Jugendlichen, die nicht vom Plan der Berufsausbildung erfaßt sind, und Schwerbeschädigten zu unterstützen und verhielten sich vielfach ablehnend. Es ist deshalb notwendig, daß dem Ministerium für Arbeit und seinen nachgeordneten Organen die Möglichkeit gegeben wird, die Betriebe, die sich den diesbezüglichen Auflagen der Organe der Arbeitsverwaltung widersetzen, zu bestrafen." 80 Der DDR-Ministerrat stimmte dem Vorschlag Chwaleks auf seiner Sitzung am 22. Januar $1953 \mathrm{zu}$ und faßte einen entsprechenden Beschluß81.

Nicht nur Betriebe und Bezirksverwaltungen, sondern auch Massenorganisationen wiesen gegenüber der Staats- und Parteiführung auf die drohende Arbeitslosigkeit von auslernenden Lehrlingen hin. So bat beispielsweise der DFD in

75 Ebenda, Aktenvermerk der Abt. Arbeitskraftlenkung vom 23. 12. 1952, S. 1. Bei den anderen Werken handelte es sich um das Stahl- und Walzwerk Riesa sowie das Metallschmelz- und Walzwerk Merseburg.

76 Ebenda, IHK Sachsen-Anhalt (Landeskammer) am 29.12. 1952 an das Ministerium für Arbeit (Abt. Arbeitskraftlenkung).

77 Ebenda, Abteilungsleiter Arndt am 16. 1. 1953 an dic IHK Sachsen-Anhalt (Landeskammer).

$78 \mathrm{BAB}, \mathrm{DC} 20$ I/3-171, Bl. 76-79, Beschlußvorlage mit Anschreiben Chwaleks vom 15. 1. 1953 an den Chef der Regierungskanzlei Fritz Geyer.

79 Das Ministerium für Arbeit ging für das Jahr 1953 von insgesamt 48000 Jugendlichen aus, die nicht vom Nachwuchsplan erfaßt wurden. Ebenda, Bl. 81, Begründung vom 14.1.1953.

80 Ebenda, Bl. 80-82, hier Bl. 80, Begründung vom 14. 1. 1953.

81 BAB, DC $20 \mathrm{I} / 3-169, \mathrm{Bl} .7$ und $178 \mathrm{f}$. 
Thale in einem Petitionsschreiben an Walter Ulbricht darum, für die rund 300 Jungfacharbeiter des Eisen- und Hüttenwerkes geeignete Arbeitsplätze innerhalb der volkseigenen Wirtschaft zu finden ${ }^{82}$. Ulbricht leitete die Anfrage an das Arbeitsministerium weiter und veranlaßte eine Überprüfung des Betriebes. Dabei sollte festgestellt werden, "wieviele Kollegen von den einzelnen Berufsgruppen zur Entlassung kommen, um in Zusammenarbeit mit dem Rat des Bezirkes für deren weitere Unterbringung Sorge zu tragen ${ }^{83}$. Nachdem der Ministerrat das Ministerium für Arbeit beauftragt hatte, eine Meldepflicht für alle unbeschäftigten Jugendlichen einzuführen, lag eine entsprechende Anweisung Ende Februar bereits vor, die an die Abteilungen für Arbeit und Berufsausbildung bei den Räten der Bezirke, Kreise und Städte adressiert wurde ${ }^{84}$. Um die statistische Erhebung zu verbessern, sollte vor allem die Zusammenarbeit mit den Berufsschulen intensiviert werden, die ihrerseits regelmäßig über die Zahl der Auszubildenden zu berichten hatten. Minister Chwalek betonte in einem Schreiben an den Staatssekretär für Berufsausbildung Wießner, daß diese "Gegenkontrolle“ letztlich unabdingbar sei, um einen exakten Überblick über die beschäftigungslosen Jugendlichen zu erhalten ${ }^{85}$. Gleichzeitig beklagte sich das Arbeitsministerium über die ausbleibende Unterstützung von seiten diverser Fachministerien: Im Zentrum der Kritik stand ganz besonders das Staatssekretariat für Kohle und Energie, das sich - so der Vorwurf - der Verantwortung für die Auszubildenden im eigenen Zuständigkeitsbereich entziehen wollte ${ }^{86}$. In der Kohlenindustrie konnten rund 1500 Lehrlinge, die vor dem Abschluß ihrer Ausbildung standen, nicht weiter beschäftigt werden, da der Arbeitskräfte- und Finanzplan 1953 eine entsprechende Vergrößerung des Beschäftigtenstandes nicht zuließ ${ }^{87}$. An diesem Einzelfall wurde erneut der grundlegende Zielkonflikt deutlich: Während das Arbeitsministerium auf eine Übernahme sämtlicher Lehrlinge drängte, sahen sich dazu die einzelnen Werksdirektoren sowie die zuständige Hauptverwaltung nicht mehr in der Lage ${ }^{88}$. Die Diskrepanz zwischen der Anzahl der Lehrlingsstellen und den angebotenen Arbeitsplätzen für ehemalige Auszubildende in den einzelnen Betrieben erklärt sich zum Teil daraus, daß vor allem Großbetriebe und Kombinate die Ausbildung für bestimmte Berufsgruppen übernommen hatten, die nicht nur zur Abdeckung des eigenen Facharbeiterbedarfs vorgesehen war. So betonte beispielsweise die VEB Maxhütte Unterwellenborn (Thüringen), daß die Lehrlinge des Werkes für

$82 \mathrm{BAB}, \mathrm{DQ} 2 / 1850$, DFD Thale am 24.1. 1953 an Walter Ulbricht. Die DFD-Kreisorganisation Quedlinburg erneuerte diese Bitte wenige Tage später. Ebenda, DFD-Kreis Quedlinburg am 4. 2. 1953 an Walter Ulbricht.

83 BAB, DQ 2/1850, Stellvertreter des Ministerpräsidenten (Sekretariat Ulbricht) am 13.2. 1953 an das Ministerium für Arbeit (Abt. Arbeitskraftlenkung).

$84 \mathrm{BAB}, \mathrm{DQ} 2 / 1839$, Anweisung vom 24. 2. 1953.

8s Ebenda, Chwalek am 10.2.1953 an Wießner.

86 Ebenda, Aktenvermerk der Abt. Arbeitskraftlenkung (Seidel) vom 25. 2. 1953.

87 Ebenda, Staatssekretariat für Kohle und Energie (Staatssekretär Fritsch) am 25. 2. 1953 an Minister Chwalek.

88 Vgl. ebenda, Staatssekretariat für Kohle und Energie (Staatssekretär Fritsch) am 27.2. 1953 an Minister Chwalek. Die Zahl der Jugendlichen, denen noch kein Arbeitsplatz im erlernten Beruf nachgewiesen werden konnte, hatte sich mittlerweile auf 957 reduziert. 
die metallurgischen Betriebe der DDR ausgebildet worden seien, „für die wir nach Ablegung ihrer Facharbeiterprüfung keine Planstellen offen haben"89.

Das Ministerium für Arbeit bereitete daraufhin eine weitere Beschlußvorlage für den Ministerrat vor, um die Absprache zwischen den beteiligten Ministerien bei der Versorgung der Lehrstelleninhaber mit Arbeitsplätzen zu verbessern ${ }^{90}$. Das Präsidium des Ministerrates verabschiedete den eingereichten Entwurf am 16. März 1953, der unter anderem die Betriebe verpflichtete, Jugendliche „für bestimmte Spezialaufgaben innerhalb ihres erlernten oder eines zweiten, verwandten Berufes zu qualifizieren“91. Weibliche Lehrlinge sollten „in jedem Falle“ im erlernten Beruf weiterbeschäftigt werden. Aufschlußreich war die Anweisung, die in den Betrieben berufsfremd eingesetzten Facharbeiter zu entlassen und statt dessen die überzähligen Jungfacharbeiter zu übernehmen. Im Gegenzug sollten die „freigestellten“ Arbeitskräfte in ihren angestammten Berufen wieder eingesetzt werden. Die Ministerien für Aufbau und für Arbeit erhielten den Auftrag, dafür zu sorgen, daß auf der Baustelle des EKO insgesamt 2400 Jugendliche zusätzlich beschäftigt wurden. Mindestens 3000 Jugendliche sollten in sechs weiteren Großbetrieben ${ }^{92}$ Arbeit finden. Damit wurden die Großbetriebe stärker in die Pflicht genommen: Sie mußten letztlich den Beschäftigtenbestand erhöhen. Es war allerdings davon auszugehen, daß die DDR-Regierung ihre Forderung nach einer Produktivitätssteigerung in den Betrieben vorerst zurückstellen würde. $\mathrm{Zu}$ diesem Zeitpunkt hatte vielmehr das Ziel, das Ansteigen von Arbeitslosigkeit zu verhindern, oberste Priorität bei den beteiligten Ressorts gewonnen. Einzelne Ministerien gaben die verabschiedete Anweisung des Ministerrates in leicht abgeänderter Form nochmals heraus ${ }^{93}$.

Das Ministerium für Hüttenwesen und Erzbergbau informierte die ihm unterstehenden volkseigenen Betriebe über die Anzahl der Lehrlinge, die im Frühjahr bzw. Herbst 1953 ihre Facharbeiterprüfung in den einzelnen Berufsgruppen ablegen würden und rief dazu auf, „durch direkte Verhandlungen mit den Lehrbetrieben von der Möglichkeit der Übernahme im Rahmen des Arbeitskräfteplanes

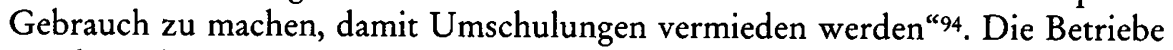
wurden gebeten, nach Ablegung der Facharbeiterprüfungen Bericht zu erstatten, "wie der freigewordene Facharbeiternachwuchs gelenkt wurde." Damit wurde der Anspruch fallengelassen, den beruflichen Nachwuchs zentral planen und lenken zu können. Statt dessen rückten die Betriebe immer mehr ins Zentrum des Geschehens. Sie hatten zuvor schon über die Einstellung von Lehrlingen maßgeblich entschieden; diese Praxis wurde vom Ministerium für Hüttenwesen und Erzbergbau nachträglich gebilligt. Während die berufliche Unterbringung der Ju-

89 BAB, DQ 2/1839, VEB Maxhütte an das Ministerium für Hüttenwesen und Erzbergbau (o.D.).

$90 \mathrm{BAB}, \mathrm{DQ} 2 / 1849$, Begründung des Ministeriums für Arbeit zur Beschlußvorlage über die Beschäftigung der im Februar 1953 die Berufsausbildung beendenden Jugendlichen (o.D.).

$91 \mathrm{BAB}, \mathrm{DC} 20 \mathrm{I} / 4-9, \mathrm{Bl} .3$ und $7 \mathrm{f}$. hier Bl. 7.

92 Dabei handelte es sich um die Großkokerei Lauchhammer, die Maxhütte Unterwellenborn, das Eisenhüttenwerk West (Calbe), die Stahl- und Walzwerke Riesa, die Mathias-Thesen-Werft und den Wohnungsbau in Wismar. Ebenda, Bl. 8.

93 Vgl. BAB, DQ 2/1839, Dienstanweisung des Ministeriums für Hüttenwesen und Erzbergbau Nr. 4 a/53 vom 28. 3. 1953.

$94 \mathrm{BAB}, \mathrm{DQ} 2 / 1847$, Rundschreiben des Ministeriums für Hüttenwesen und Erzbergbau vom 10. 2. 1953 (korrigiert auf den Stand vom 19. 3. 1953). 
gendlichen, die ihre Ausbildung im Frühjahr beendeten, weitgehend gesichert werden konnte, schien sich für den Herbst ein erhebliches Versorgungsproblem zu ergeben. Die Hauptverwaltung Kohle rechnete mit etwa 130 Lehrlingen, die nach den Prüfungen des Frühjahrs nicht unterzubringen waren, ein halbes Jahr später aber schon mit 237195. Der Handlungsdruck auf die Fachministerien sowie die Betriebe würde demzufolge im Laufe des Jahres 1953 weiter ansteigen.

Das Ministerium für Hüttenwesen und Erzbergbau ermittelte für die Stahlund Walzwerke 1626 Jungfacharbeiter, die bis zum Ende des Jahres nicht vom auszubildenden Betrieb übernommen wurden ${ }^{96}$. Vor allem die Großbetriebe sahen sich, wie bereits erwähnt, nicht in der Lage, sämtliche Lehrlinge einzustellen. Die Maxhütte meldete etwa einen Überhang von insgesamt 304 angehenden Facharbeitern, das Stahl- und Walzwerk Riesa 343, das Edelstahlwerk Döhlen 113 und das Mansfeld Kombinat „Wilhelm Pieck“ 13397. Die Mehrzahl der Entlassungen drohte im Herbst (1394), während die Anzahl der Jugendlichen, die ihre Lehre abgeschlossen hatten und nicht übernommen werden konnten, im Frühjahr sehr viel niedriger lag (232) ${ }^{98}$. Von den übrigen Fachministerien lagen zu diesem Zeitpunkt keine vergleichbaren Berichte vor. Das Ministerium für Arbeit bemühte sich in der Folgezeit die Ministerien bzw. Staatssekretariate dazu zu bewegen, den vorgegebenen Arbeitskräfteplan nach oben zu korrigieren. Dadurch sollte sichergestellt werden, daß die Jugendlichen nicht in die Arbeitslosigkeit entlassen, sondern von anderen Betrieben zusätzlich eingestellt wurden. Da jedoch die Fachministerien oftmals verspätet und unzureichend über die Verteilung der überzähligen Jungfacharbeiter berichteten, konnte das Arbeitsministerium nur einen ersten vorläufigen Überblick zusammenstellen 99 .

Den Zwischenbericht legte Staatssekretärin Malter dem Ministerrat auf der Sitzung am 28. Mai 1953 vor. Dieser übernahm weitgehend den vom Arbeitsministerium ausgearbeiteten Maßnahmenkatalog. Von zentraler Bedeutung war die Entscheidung, den Fachministerien und Staatssekretariaten „die volle Verantwortung für die Unterbringung ihrer auslernenden Jugendlichen " zu übertragen ${ }^{100}$. Darüber hinaus sollten die „tatsächlichen Überhänge [...] lückenlos“ dem Ministerium für Arbeit gemeldet werden, das wiederum Vorschläge für die Verteilung der Lehrlinge, die noch kein Beschäftigungsverhältnis nach Beendigung der Ausbildungszeit in Aussicht hatten, ausarbeiten und nach Absprache mit der Staatlichen Plankommission dem Ministerrat erneut vorlegen sollte. Mit der zunehmenden Militarisierung von Staat und Gesellschaft 1952/53 rückten zwei neue Varianten in den Mittelpunkt der Überlegungen, um die drohende Jugendarbeitslosigkeit abzuwenden: der Aufbau bewaffneter Organe sowie der „Dienst für Deutsch-

${ }^{95} \mathrm{BAB}, \mathrm{DQ} 2 / 1839$, HV Kohle am 1.4. 1953 an die Abt. Planinspektion und Materialbilanzierung, S. 2.

96 Ebenda, Ministerium für Hüttenwesen und Erzbergbau (Staatssekretär Goschütz) am 18. 5. 1953 an das Ministerium für Arbeit, Anlage 1, Bl. 4.

97 Ebenda, Bl. 1 und 3.

${ }^{98}$ Ebenda, Anlage 3: Zusammenstellung des Ministeriums für Hüttenwesen und Erzbergbau (Abt. Arbeit) vom 15. 5. 1953, S. 6.

99 Vgl. BAB, DQ 2/1570, Zwischenbericht des Ministeriums für Arbeit vom 27. 5. 1953.

$100 \mathrm{BAB}, \mathrm{DC} 20 \mathrm{I} / 3-190, \mathrm{Bl} .4$. 
Tabelle 22: Überblick über die Zabl der auslernenden Jugendlichen (Stand: Mai 1953)

\begin{tabular}{lcc}
\hline & $\begin{array}{c}\text { auslernende } \\
\text { Jugendliche } \\
\text { insgesamt }\end{array}$ & $\begin{array}{l}\text { Anzahl der Jugend- } \\
\text { lichen, die nicht } \\
\text { ubernommen } \\
\text { werden können }\end{array}$ \\
\hline Ministerium für Hüttenwesen und Erzbergbau & 5025 & 1300 \\
Ministerium für Leichtindustrie & 10800 & 1063 \\
Ministerium für Post- und Fernmeldewesen & 1300 & - \\
Ministerium für Aufbau & 4467 & - \\
Ministerium für Schwermaschinenbau & 10656 & 4750 \\
Ministerium für Eisenbahnwesen & 7500 & 3800 \\
Ministerium für Allgemeinen Maschinenbau & 12100 & 5200 \\
Staatssekretariat für Kohle & 3300 & 1410 \\
Staatssekretariat für Energie & 1486 & 800 \\
Staatssekretariat für Chemie & 4543 & 1465 \\
Staatssekretariat für Nahrungs- und & 1860 & 250 \\
Genußmittelindustrie & 91 & - \\
Staatssekretariat für Schiffahrt & 63128 & 20038 \\
\hline Insgesamt & & \\
\hline
\end{tabular}

Quelle: BAB, DQ 2/1570, Zwischenbericht des Ministeriums für Arbeit vom 27. 5. 1953, S. 7 f.

land"101. Da der stark expandierende Sicherheitsapparat der DDR einen enormen Arbeitskräftebedarf mit sich brachte, schien sich auf diesem Wege eine Lösung der arbeitsmarktpolitischen Schwierigkeiten abzuzeichen. Doch schon frühzeitig mußte das Arbeitsministerium erkennen, daß dadurch kurz- und mittelfristig eine spürbare Entlastung nicht zu erwarten war, zumal der am 9. Juni 1953 von der SED-Führung verkündete „Neue Kurs“ bereits das Ende der kurzlebigen Organisation „Dienst für Deutschland“ bedeutete ${ }^{102}$. Auch das Ministerium für Arbeit konnte angesichts der geschilderten Ausgangslage keine Patentlösungen entwickeln und sprach sich statt dessen unverbindlich dafür aus, auch in Zukunft beschäftigungslose Jugendliche verstärkt bei Großbetrieben bzw. -projekten einzusetzen. Die konkrete Umsetzung dieses Vorschlages blieb somit nach wie vor von der Kooperationsbereitschaft der Betriebs- und Werksleiter abhängig.

\section{Arbeitseinsatz von Strafgefangenen}

Der Arbeitsverwaltung war es offenbar rasch gelungen, in Zusammenarbeit mit der Justizverwaltung einen Großteil der Gefängnisinsassen für den Arbeitseinsatz zu mobilisieren. In Sachsen-Anhalt waren von insgesamt 2420 Strafgefangenen Ende 19491682 beschäftigt, 853 außerhalb und 829 innerhalb der Haftanstalten ${ }^{103}$. Dabei mußte jedoch berücksichtigt werden, daß 1979 Gefangene, die sich

101 Vgl. Buddrus, Die Organisation „Dienst für Deutschland“.

102 Ebenda, S. 212 f. Zur Einschätzung des Arbeitsministeriums: BAB, DQ 2/1570, Entwurf des Ministeriums für Arbeit vom 12.6. 1953.

103 BAB, DQ 2/1870, Ministerium für Arbeit und Gesundheitswesen der Landesregierung SachsenAnhalt am 19. 1. 1950 an das Ministerium für Arbeit und Gesundheitswesen (HA Arbeit). 
am 1. September 1949 in Untersuchungshaft befanden, für den Arbeitseinsatz nicht in Frage kamen. Ein grundlegendes Problem bestand darin, daß sich einige Anstalten nicht in unmittelbarer Nähe zu wirtschaftlichen Großprojekten befanden und deshalb eine Beschäftigung der Häftlinge nicht zustande kommen konnte. So saßen beispielsweise in der Strafanstalt Torgau rund 500 Gefangene ein, „die mangels dort vorhandener Industrie zum großen Teil brach liegen“ ${ }^{104}$. Nachdem für die dortigen Strafgefangenen keine Beschäftigungsmöglichkeiten im Raum Torgau gefunden werden konnten, zog das Ministerium der Justiz die Verlegung von Häftlingen in eine andere Anstalt in Erwägung, die im Einzugsbereich eines Industrieschwerpunktes lag ${ }^{105}$. Darüber hinaus gab es nach wie vor Vorbehalte auf seiten der Unternehmer, Strafgefangene zeitweise zu beschäftigen. Selbst Werksleiter von volkseigenen Betrieben zeigten sich „erst dann zugänglich [...], wenn sie von oben her eine entsprechende Anweisung" erhielten, so die generelle Einschätzung Max Fechners ${ }^{106}$, der anläßlich einer Tagung mit Vertretern der Landesjustizministerien sowie des Ministeriums für Industrie erneut die „erzieherische“ Funktion des Strafvollzugs betonte107. Das Justizministerium, das sich mittlerweile in einer Kontroverse mit dem Ministerium des Innern über die $\mathrm{Zu}$ ständigkeit des Strafvollzugs befand ${ }^{108}$, versuchte den FDGB als Verbündeten zu gewinnen. Dieser wurde nämlich gebeten, eine betriebliche Aufklärungsarbeit zu unterstützen, um das Arbeitsangebot für Strafgefangene innerhalb und außerhalb der Gefängnismauern insgesamt zu verbessern ${ }^{109}$. Sowohl die Arbeits- als auch die Justizverwaltung hatten offenbar kein Interesse daran, daß Bewährungsarbeiter bei der Wismut AG eingesetzt wurden. Die sächsische Landesverwaltung forderte eine entsprechende Anweisung an die übrigen Länder ${ }^{110}$. Dadurch sollte verhindert werden, daß die soziale Lage im Erzbergbaugebiet, die 1950 immer noch angespannt war, erneut verschärft wurde. Dagegen sprach sich das Berliner Arbeitsministerium dafür aus, Verhandlungen mit der sächsischen Landesjustizverwaltung aufzunehmen, um den Einsatz von Strafgefangenen im Zwickauer Steinkohlenbergbau systematisch auszudehnen ${ }^{111}$. Um Fehlvermittlungen zu vermeiden,

104 Ebenda.

105 Ebenda.

106 SAPMO, DY 34, 18/-/530, Notiz Fechners vom 4. 3. 1950.

$107 \mathrm{BAB}, \mathrm{DQ} 2 / 1870$, Niederschrift über die Arbeitsplanungskonferenz der HA Strafvollzug und Anstaltsverwaltung des Ministeriums der Justiz am 9.6. 1950, S. 2. Fechner kündigte in dem Zusammenhang die Einführung eines sogenannten klassifizierten Strafvollzugs an: Dabei sollten z.B. die „schwersten Arbeiten [...] von den schwersten Verbrechern ausgeführt werden“. Ebenda, S. 3.

108 Vgl. dazu Wentker, Justiz in der SBZ/DDR, S. 369-398.

109 SAPMO, DY 34, 18/-/530, Aktennotiz der FDGB-Abt. Arbeit und Sozialpolitik (Facius) vom 17. 3. 1950 an Kirchner und Kummerlöw.

$110 \mathrm{BAB}, \mathrm{DQ} 2 / 1870$, Ministerium für Arbeit und Gesundheitswesen der Landesregierung Sachsen am 13. 4. 1950 an das Ministerium für Arbeit und Gesundheitswesen (HA Arbeit). Das Ministerium der Justiz und das Ministerium für Arbeit und Gesundheitswesen wiesen die mecklenburgische Landesverwaltung im Juni 1950 darauf hin, daß für den Uranbergbau keine Bewährungsarbeiter zugewiesen werden. Vgl. ebenda. Die Durchsetzung dieser Anweisung gelang auch innerhalb der Justizverwaltung nicht vollständig. So gingen beim Arbeitsamt Aue im Herbst 1950 "täglich" Schreiben von Oberstaatsanwaltschaften und Amtsgerichten ein, in denen festgelegt wurde, „daß die zugestandene Bewährungsarbeit bei der Wismut AG abzuleisten ist“. BAB, DQ 2/1870, Hauptabteilungsleiter Litke am 11. 10. 1950 an das Ministerium der Justiz.

$111 \mathrm{BAB}, \mathrm{DQ} 2 / 2132$, Arbeitsamt Zwickau am 13.6. 1950 an die Industrieverwaltung Steinkohle in Zwickau. 
sollten auch in diesem Fall zunächst einmal ärztliche Voruntersuchungen durchgeführt werden, in welchen die Bergbautauglichkeit der ausgewählten Häftlinge zu überprüfen war.

Im Sommer 1950 verständigten sich das Justiz- und das Arbeitsministerium darauf, den Arbeitseinsatz von Strafgefangenen in „zentral erfaßten Arbeitsvorkommen vorzunehmen". Vorgesehen war eine Aufteilung in drei Gruppen: bergbautaugliche Bewährungsarbeiter für Untertagearbeit, für Übertagearbeit sowie für den Einsatz in volkseigenen Betrieben ${ }^{112}$. Wie unzureichend die Absprachen zwischen der Arbeits- und Justizverwaltung auf lokaler Ebene mitunter waren, zeigt ein Schreiben des Arbeitsamtes Torgau, das die Notwendigkeit unterstrich, vor jedem Gefangenen-Arbeitskommando das zuständige Arbeitsamt zu informieren ${ }^{113}$. Die besondere wohnliche Unterbringung der Bewährungsarbeiter in eigens abgesicherten Barackenlagern rückte dagegen erst relativ spät in den Mittelpunkt der Diskussion. So wurde etwa das Mansfeld Kombinat, das Interesse am verstärkten Arbeitseinsatz von Strafgefangenen angemeldet hatte, aufgefordert, Planungsunterlagen über die Errichtung weiterer Gefangenenbaracken beim Ministerium für Planung einzureichen ${ }^{114}$. Da diese im Sommer 1950 noch nicht vorlagen, ließ sich auch die Anzahl der dort beschäftigten Häftlinge nicht erhöhen. Erneut erwiesen sich somit die sicherheitspolitischen Bedenken als Hindernis für die Ausdehnung der Bewährungsarbeit in den volkseigenen Betrieben.

Um die Beschäftigung von Strafgefangenen auszuweiten, sprach sich das Ministerium der Justiz im Juni 1950 erneut für die Verlagerung von Betriebsteilen nahe gelegener Unternehmen in die Haftanstalten aus. Darüber hinaus sollte die $\mathrm{Zu}$ sammenarbeit zwischen den Gefängnisleitungen und den zuständigen Mitarbeitern der Arbeitsämter verbessert werden. Gleichzeitig beabsichtigte das Justizministerium, eine „Verordnung über die Einplanung des Arbeitspotentials der Strafanstalten" herauszugeben ${ }^{115}$. Als einziges Ministerium sprach sich das Ministerium für Industrie gegen dieses Vorhaben aus und verwies zur Begründung darauf, daß „die ideologische Einstellung der Betriebe noch unterschiedlich" sei. $\mathrm{Da}$ in dieser Frage zunächst keine Übereinstimmung erzielt werden konnte, wurde die Ausarbeitung des Verordnungsentwurfs zunächst zurückgestellt. Das bedeutete jedoch keineswegs, daß der Arbeitseinsatz von Strafgefangenen grundsätzlich in Frage gestellt wurde. Im Gegenteil: Die Veröffentlichung des Gesetzes der Arbeit Ende April 1950 hatte eine rege Propagandatätigkeit zugunsten der Bewährungsarbeit in der DDR-Presse ausgelöst. Dadurch sollte die Unterstützung aller Ministerien, Massenorganisationen und vor allem der Betriebsleiter gewonnen werden. In der Berliner Zeitung wies etwa K. Ambrée auf den Erziehungsaspekt der Bewährungsarbeit hin und unterstrich, daß die Bestimmungen des neu erlassenen Arbeitsgesetzes auch auf die Bewährungsarbeiter Anwendung finden müßten. Daraus zog der Verfasser des Artikels die Konsequenz, daß ein Gefange-

112 BAB, DQ 2/1870, gemeinsames Schreiben des Ministeriums der Justiz und des Ministeriums für Arbeit und Gesundheitswesen vom Juni 1950 an die mecklenburgische Justizverwaltung.

113 Ebenda, Amt für Arbeit und Sozialfürsorge am 13.6. 1950 an das Ministerium für Arbeit und Gesundheitswesen der Landesregierung Sachsen-Anhalt.

${ }^{11+}$ Ebenda, Aktennotiz Krügers vom 17.6. 1950.

115 Ebenda, Aktenvermerk der Abt. Arbeitskraftlenkung vom 24. 6. 1950. 
ner, der sich in einem Beschäftigungsverhältnis befand, unter anderem „Anspruch auf volle Entlohnung" habe ${ }^{116}$. Der Erziehungsgedanke der Bewährungsarbeit, den sowohl das Justiz- als auch das Arbeitsministerium ${ }^{117}$ stets betont hatten, blieb allerdings nicht unwidersprochen. Ende August erschien in der Berliner Zeitung ein Beitrag, der sich explizit gegen eine Gleichbehandlung dieser Personengruppe in arbeitsrechtlicher Hinsicht aussprach ${ }^{118}$. Das Arbeitsministerium unterstrich in der Folgezeit mehrmals die unterschiedliche Behandlung von Bewährungsarbeitern und Strafgefangenen mit einer langjährigen Haftstrafe in arbeitsrechtlicher und tarifrechtlicher Hinsicht. Dabei konnte sich das Ministerium auf die gemeinsame Dienstanweisung der Justiz- und Arbeitsverwaltung vom 1. September 1947119 stützen, die zwar keine expliziten Angaben zur tariflichen Einstufung enthielt, dafür aber eine deutliche Trennung zwischen den beiden Gefangenengruppen vollzog. Daraus leitete letztlich das Arbeitsministerium die unterschiedliche arbeitsrechtliche Stellung der Bewährungsarbeiter auf der einen und der langfristig Verurteilten auf der anderen Seite $\mathrm{ab}^{120}$.

Angesichts des steigenden Arbeitskräftebedarfs in den wirtschaftlichen Schwerpunktbetrieben unternahmen Mitarbeiter des Arbeitsministeriums des öfteren Dienstreisen zu den im Aufbau befindlichen Werken, um zusätzliche Beschäftigungsmöglichkeiten für Strafgefangene zu sondieren. Anläßlich einer Reise zum EKO nach Fürstenberg am 22. August 1950 wurde sogar der Vorschlag erörtert, in unmittelbarer Nähe zum EKO eine Gefangenenanstalt zu errichten, „in die ganze Produktionszweige des neuen Werkes gelegt werden können" 121 . Da der Bau der Wohnstadt Fürstenberg und die Errichtung der Hochofenanlage zu diesem Zeitpunkt weit hinter den Planungen zurücklagen, wurde dieser Vorstoß, der die ohnehin angespannte Versorgung mit Baumaterialien weiter verschlechtert hätte, jedoch nicht aufgegriffen. Der anhaltende Bedarf an zusätzlichen Arbeitskräften hatte im übrigen zur Folge, daß der Einsatz von Strafgefangenen im Erzgebirge, der ursprünglich ausdrücklich ausgeschlossen worden war, zumindest zeitweise toleriert wurde ${ }^{122}$.

Einzelne Arbeitsämter bewerteten im Frühjahr 1951 die Einführung der Bewährungsarbeit durchaus als Erfolg. So verwies das Arbeitsamt Leipzig darauf, daß „der größte Teil der Bewährungsarbeiter nach Beendigung der Arbeitsauflage

116 K. Ambrée: „Gilt das Gesetz der Arbeit für Strafgefangene?“, in: ,Berliner Zeitung vom 20. 8. 1950. Zitiert nach: BAB, DQ 2/1372. In etwas abgeänderter Form erschien der Artikel auch in der ,Tribüne‘. Vgl. Kurt Ambrée: „Die arbeitsrechtliche Stellung der Strafgefangenen“, in: ,Tribüne“ vom 22.-24. 8. 1950.

11 Dabei wies das Arbeitsministerium allerdings auf die unterschiedlichen arbeitsrechtlichen Bestimmungen für Bewährungsarbeiter und Strafgefangene hin, die in Arbeitskolonnen in Betrieben und Bauvorhaben eingesetzt wurden. Während die Bewährungsarbeiter den Regelungen des Arbeitsgesetzes unterworfen waren, galt dies nicht für die zweite Gefangenengruppe. Entscheidend war somit die Einstufung als Bewährungsarbeiter. Vgl. Hermann Kienast: Gilt das Gesetz der Arbeit für Strafgefangene, in: Arbeit und Sozialfürsorge 5 (1950).

118 Dr. G. Berger: „Mißverstandener humanitärer Strafvollzug“, in: ,Berliner Zeitung vom 29.8. 1950.

119 Arbeit und Sozialfürsorge 2 (1947), S. 376-378.

$120 \mathrm{BAB}, \mathrm{DQ} 2 / 1373$, Vermerk Heisigs vom 29. 8. 1950.

$121 \mathrm{BAB}, \mathrm{DQ} 2 / 2152$, Bericht der HA III vom 23. 8. 1950, S. 2.

122 So z. B. beim Ausbau der Gleisanlagen im Kreis Aue. Vgl. SächsHStA, Landesregierung Sachsen, Ministerium für Arbeit und Sozialfürsorge, Bd. 343, Bericht des Arbeitsamtes Aue vom 16. 4. 1951 an das Ministerium für Industrie, Arbeit und Aufbau (HA Arbeit) in Dresden. 
in den Bewährungsarbeiterbetrieben, die Schwerpunkte unserer Wirtschaft sind, verbleiben" ${ }^{123}$. In dem Zusammenhang konnte das Arbeitsamt 21 Betriebe nennen, die sich in der Vergangenheit stets bereit erklärt hatten, Arbeitsplätze zur Verfügung zu stellen. Dennoch zeigen die überlieferten Statistiken, daß das quantitative Ausmaß der Bewährungsarbeit unter dem Gesichtspunkt der Gewinnung zusätzlicher Arbeitskräfte bescheiden blieb. Nach Angaben des Leipziger Arbeitsamtes waren innerhalb eines Jahres insgesamt 580 Bewährungsarbeiter (410 Männer, 170 Frauen) durch die Justizbehörde zugewiesen worden. Davon seien allerdings 82 Personen infolge von Arbeitsbummelei und Nichtantritt, sieben wegen Krankheit und 32 aufgrund nachträglicher Annullierung nicht zum Arbeitseinsatz gekommen ${ }^{124}$. Das Arbeitsamt Dresden meldete im selben Zeitraum 378 Bewährungsarbeiter, die in Betrieben des Arbeitsamtsbezirks eingestellt wurden, von denen wiederum 14 Personen infolge von Arbeitsbummelei oder erneuter Straffälligkeit sowie zwölf aufgrund von Krankheit letztlich nicht beschäftigt werden konnten ${ }^{125}$.

Das Sekretariat des ZK der SED befaßte sich zwar am 20. August $1951 \mathrm{mit}$ dem Arbeitseinsatz von Strafgefangenen und diskutierte offensichtlich einen entsprechenden Verordnungsentwurf, den vermutlich das Arbeitsministerium eingereicht hatte. Die SED-Führung vermied allerdings eine abschließende Stellungnahme und beauftragte die ZK-Abteilung Staatliche Verwaltung zu prüfen, inwieweit in die geplante Verordnung eine Bestimmung aufgenommen werden könne, „daß die Organe des Innenministeriums das Recht haben, Arbeitslager zur Ableistung von Arbeit zu errichten" ${ }^{126}$. Mittlerweile hatte sich die Zuständigkeit für den Strafvollzug grundlegend geändert: Bereits Ende 1950 war die Entscheidung über die Übertragung des Strafvollzugs von der Justiz- auf die Innenverwaltung getroffen worden ${ }^{127}$. Die Ausarbeitung einzelner Durchführungsbestimmungen zog sich noch bis Anfang 1952 hin. Anhand der oben angeführten Berichte einzelner Arbeitsämter läßt sich zeigen, daß das Justizministerium beim Einsatz der Bewährungsarbeiter auch noch im Frühjahr 1951 entscheidend mitgewirkt hat ${ }^{128}$. Offensichtlich zog sich die Übertragung dieses Zuständigkeitsbereiches auf das Ministerium des Innern noch etwas länger hin.

Einzelne Betriebe beklagten sich in zunehmenden Maße darüber, daß zugewiesene Bewährungsarbeiter unentschuldigt der Arbeit fernblieben, und forderten die Einführung des Leistungslohnprinzips auch in diesem Bereich. Nicht nur die Lohnhöhe, sondern auch die Bewährungsfrist sollte von der "nachgewiesenen Arbeitsmoral“ und von der „Erfüllung der Arbeitsnorm“ abhängig gemacht werden,

${ }^{123}$ SächsHStA, Landesregierung Sachsen, Ministerium für Arbeit und Sozialfürsorge, Bd. 343, Arbeitsamt Leipzig am 12.5. 1951 an das Ministerium für Industrie, Arbeit und Aufbau in Dresden, S. 2.

${ }^{124}$ Ebenda. Der Berichtszeitraum ging vom 1. 4. 1950 bis zum 31. 3. 1951.

125 SächsHStA, Landesregierung Sachsen, Ministerium für Arbeit und Sozialfürsorge, Bd. 343, Arbeitsamt Dresden am 15. 5. 1951 an das Ministerium für Industrie, Arbeit und Aufbau in Dresden, S. 2.

126 SAPMO, DY 30/J IV 2/3/225, B1. 12.

127 Vgl. dazu Wentker, Justiz in der SBZ/DDR, S. 380-388.

128 So berichtete etwa das Arbeitsamt Leipzig am 12.5. 1951: „Über die Zusammenarbeit mit den Justizbehörden und Strafvollzugsanstalten ist nichts besonderes Nachteiliges zu melden." SächsHStA, Landesregierung Sachsen, Ministerium für Arbeit und Sozialfürsorge, Bd. 343. 
so der Vorschlag der VVB Schiefer ${ }^{129}$. Um die Verteilung der Bewährungsarbeiter besser kontrollieren zu können, nannte das Ministerium der Justiz nach Absprache mit dem Ministerium für Arbeit zwei wirtschaftliche Schwerpunktvorhaben, die in Zukunft für den Arbeitseinsatz der genannten Personengruppe in Frage kommen sollten ${ }^{130}$. Die Zuteilung sollte somit nicht mehr den von den Betrieben gemeldeten Bedarf abdecken, sondern hatte sich statt dessen an übergeordneten sicherheitspolitischen Aspekten zu orientieren. Gegenüber den Landesregierungen von Brandenburg und Mecklenburg nannte das Justizministerium die Baustelle der Bau-Union Fürstenberg sowie das dortige EKO als Arbeitsschwerpunkte mit einem Gesamtbedarf von insgesamt 450 bis 800 Personen ${ }^{131}$. Auf die Berichte über Arbeitsbummelei einzelner Bewährungsarbeiter reagierte das Ministerium der Justiz umgehend: Das Ministerium für Arbeit wurde aufgefordert, die Arbeitsämter zur strikten Einhaltung der gemeinsamen Richtlinien vom 1. September 1947 zu veranlassen ${ }^{132}$. So sollte etwa im Fall von Arbeitsverweigerung die Bewährungsarbeit widerrufen und in eine Haftstrafe umgewandelt werden ${ }^{133}$. Während die Justiz- und Arbeitsverwaltung versuchten, den Arbeitseinsatz von Bewährungsarbeitern auf bestimmte Schwerpunktbetriebe zu konzentrieren, und am 2. Oktober eine entsprechende Anordnung an die Landesregierungen herausgaben, konnte der Vorstoß zur Einführung des Leistungslohnes nicht verwirklicht werden ${ }^{134}$. Die getroffene Maßnahme galt jedoch nicht für Bewährungseinsätze unter zwei Monaten; in solchen Fällen konnten sich die Betriebe auch weiterhin um die Zuweisung von Bewährungsarbeitern bemühen. Generelle Ziele waren neben dem genannten Kontrollaspekt - etwas überraschend - der betriebliche Rentabilitätsgedanke: „Diese Regelung war unbedingt erforderlich, um die bisherige wenig kontrollierbare Art des Einsatzes zu vermeiden, der dem Betrieb meist mehr Kosten und Ärger verursachte, als Nutzen dabei heraussprang." ${ }^{135}$

Die Bewährungsarbeiter und die Strafgefangenen im Außeneinsatz vergrößerten zwar den Arbeitskräftebestand der einzelnen Betriebe, stellten jedoch keine konstante Planungsgröße dar. Aufgrund der zeitlich eng begrenzten Arbeitseinsätze mußte für diese Personengruppe eine nicht unbeträchtliche Fluktuationsrate in Rechnung gestellt werden. Die Unsicherheiten auf seiten der Betriebsleitungen vergrößerten sich noch zusätzlich durch Amnestieerlasse, welche die Entlassung von Strafgefangenen auch aus dem Arbeitseinsatz zur Folge haben konnte. Vor allem kleine und mittlere Betriebe sahen deshalb die Aufrechterhaltung der Produktion gefährdet. So hatten beispielsweise die Basaltwerke Vacha und Masbach (Thüringen) insgesamt 30 Bewährungsarbeiter und Strafgefangene in Arbeitskommandos, die durch die am 7. Oktober 1951 erlassene Amnestie sofort entlas-

129 BAB, DQ 2/1871, VVB Schiefer Steinach (Thüringen) am 3. 9. 1951 an die Ministerium für Justiz, Schwerindustrie und Arbeit.

130 Ebenda, Ministerium der Justiz am 15. 9. 1951 an die Landesregierungen Brandenburg und Mecklenburg.

131 Ebenda.

132 BAB, DQ 2/1871, Ministerium der Justiz (Dr. Gentz) am 17.9. 1951 an das Ministerium für Arbeit.

133 Ebenda, Hauptabteilungsleiter Dr. Gentz am 18. 9. 1951 an die VVB Schiefer in Steinach (Thüringen).

${ }_{134}$ Ebenda, Abt. Arbeitskraftlenkung am 5. 11. 1951 an die VVB Schiefer in Steinach (Thüringen).

${ }^{135}$ Ebenda, Abt. Arbeitskräftelenkung (Heisig) am 6. 11. 1951 an die VVB Ziegel in Halle/Saale. 
sen werden mußten ${ }^{136}$. Die ungünstige geographische Lage der Betriebe, die sich im grenznahen Gebiet befanden, und die nahe gelegene Kaliindustrie erschwerten die Gewinnung neuer Arbeitskräfte enorm, so daß die Betriebsleitungen nur drei Beschäftigte in der Steingewinnung melden konnten. Die Produktionsleistungen gingen rapide zurück, und für das Jahr 1951 wurde bereits mit einem Rückstand von zehn Prozent gerechnet. Das Ministerium für Schwerindustrie, das von den Werken hilfesuchend eingeschaltet worden war, stellte daraufhin beim Ministerium für Arbeit den Antrag, „Maßnahmen zur Beseitigung des Arbeitskräftemangels“ durchzuführen ${ }^{137}$. Das thüringische Ministerium für Wirtschaft und Arbeit richtete an das Berliner Arbeitsministerium die Bitte, „nochmals mit dem Ministerium des Innern - HV Volkspolizei - Rücksprache zu nehmen und zu erreichen, daß wieder Strafgefangene diesen Betrieben zugewiesen werden“"138. Das Ministerium für Arbeit leitete die Anfrage wiederum an das zuständige Fachministerium weiter: das Staatssekretariat Chemie, Steine und Erden ${ }^{139}$. Letztlich mußten die Betriebe ihren Arbeitskräftemangel eigenständig beheben. Dazu waren sie im übrigen durch die mehrfach erwähnte "Verordnung über die Aufgaben der Arbeitsverwaltungen und über die Lenkung der Arbeitskräfte" vom 12. Juli 1951 aufgefordert worden.

Das Ministerium der Justiz und das Ministerium für Arbeit strebten Anfang 1952 die Überarbeitung der gemeinsamen Richtlinien vom 1. September 1947 an ${ }^{140}$. Dabei sollte nicht nur der geänderten Amtsbezeichnung Rechnung getragen werden, sondern auch der bereits eingespielten Praxis, Bewährungsarbeiter vor allem in Schwerpunktbetrieben einzusetzen. Bei den Beratungen kristallisierte sich rasch heraus, daß eine allgemein gehaltene Verordnung sowie eine erste Durchführungsbestimmung vom Justizministerium erarbeitet und erlassen werden sollte, während das Arbeitsministerium für die arbeitsrechtlichen Bestimmungen zuständig war, die in Form einer zweiten Durchführungsbestimmung herausgegeben werden sollten ${ }^{141}$. Ein erster Entwurf der zuständigen Abteilung Arbeitsrecht im Ministerium für Arbeit lag am 15. Januar 1952 vor ${ }^{142}$. Demnach erfolgte die Entlohnung bei einer Gruppe von Strafgefangenen, die in der ersten Durchführungsverordnung noch genannt werden mußte, nach den Lohnsätzen der geltenden Kollektivverträge. Darüber hinaus unterlagen die Gefangenen aber gesonderten arbeitsrechtlichen Bestimmungen: So durften an sie Deputate, die den Arbeitern und Angestellten zustanden, nicht ausgegeben werden. Gleichzeitig konnten allerdings „für besonders gute Arbeit" den Strafgefangenen Sonderprämien gewährt werden. Der Lohn sollte den einzelnen Strafgefangenen auf einem eigens dafür eingerichteten Konto gutgeschrieben werden, über das der

136 BAB, DQ 2/2150, Ministerium für Schwerindustrie (HV Steine und Erden) am 17.12. 1951 an das Ministerium für Arbeit (Abt. Arbeitskräftelenkung).

137 Ebenda.

$138 \mathrm{BAB}, \mathrm{DQ} 2 / 2150$, Aktenvermerk des Ministeriums für Arbeit (Abt. Arbeitskraftlenkung) vom 3.1. 1952.

139 Ebenda, Abt. Arbeitskraftlenkung am 3.1. 1952 an das Staatssekretariat Chemie, Steine und Erden.

$140 \mathrm{BAB}, \mathrm{DQ} 2 / 1871$, Entwurf der beiden Ministerien vom 2.1. 1952.

141 BAB, DQ 2/1373, Vermerk von Abteilungsleiter Schaum vom 17. 1. 1952.

142 Ebenda, 2. Durchführungsbestimmung zur Verordnung über den Arbeitseinsatz von Strafgefangenen (Entwurf). 
Betreffende nach der Haftentlassung verfügen konnte. Vom Lohn wurden Haftsowie Unterstützungskosten für Familienangehörige abgezogen. Die gesetzlichen Arbeitsschutzbestimmungen sollten im übrigen auch für Strafgefangene im Arbeitseinsatz gelten. Der DDR-Ministerrat beschloß am 3. April die „Verordnung über die Beschäftigung von Strafgefangenen "143, die auf einem Entwurf des Justizministeriums basierte ${ }^{144}$. In arbeitsrechtlicher Hinsicht war hervorzuheben, daß die Strafgefangenen mit Ausnahme der Deputate den übrigen Beschäftigten gleichgestellt wurden: Das betraf die Regelungen der Entlohnung sowie des Arbeitsschutzes ${ }^{145}$. Im Gesetzblatt erschien die Verordnung wenige Tage später, nämlich am 8. April $1952^{146}$.

Ende Februar 1952 forderte das Ministerium für Arbeit die fünf Landesregierungen in einem Rundschreiben auf, Arbeitsschwerpunkte für weibliche Bewährungsarbeiter zu schaffen ${ }^{147}$. Außerdem wurde den zuständigen Landesministerien ein "Organisationsplan" für den Arbeitseinsatz von Bewährungsarbeitern vorgelegt, der zusammen mit dem Ministerium der Justiz ausgearbeitet worden war und die Bedarfsmeldung sowie die Zuteilung genau regeln sollte. Bewährungsarbeiter, die zu einer Bewährungszeit von über zwei Monaten verurteilt worden waren, wurden im Sommer 1952 in folgenden Schwerpunktbetrieben eingesetzt: Maxhütte (Thüringen), Bleierzgruben bei Freiberg (Sachsen), Mansfeld Kombinat (Sachsen-Anhalt), EKO und Bau-Union in Fürstenberg (Brandenburg). Bewährungsarbeiter mit einer Bewährungszeit bis zu zwei Monaten sollten dagegen in „Kleineinsätzen“ auf den landwirtschaftlichen volkseigenen Gütern (VEG) untergebracht werden ${ }^{148}$. Da sich offensichtlich zahlreiche Betriebe an die Hauptverwaltung Deutsche Volkspolizei, das Ministerium für Arbeit oder das Ministerium der Justiz mit der Bitte um Zuweisung von Strafgefangenen gewandt hatten, wurden in der Folgezeit weitere Großbetriebe in den Empfängerkreis aufgenommen ${ }^{149}$. Aus sicherheitspolitischen Erwägungen heraus blieb die Anzahl der Betriebe aber insgesamt sehr beschränkt. Von seiten der Generalstaatsanwaltschaft kam Ende Oktober 1952 sogar der Vorschlag, „Gefangenenabteilungen bei großen VEB zu errichten, in denen diese getrennt von anderen Werktätigen beschäftigt werden und auch unmittelbar untergebracht sind"150. Das hätte letztendlich zur Folge gehabt, daß der Bau von Strafvollzugsanstalten hinfällig geworden wäre.

143 BAB, DC 20 I/3-101, Bl. 4

144 Ebenda, Bl. 70-72. In der Begründung zum Verordnungsentwurf verwies das Ministerium der Justiz darauf, daß bereits am 31. 1. 1951 eine Vereinbarung mit dem Ministerium für Schwerindustrie und dem Ministerium des Innern über den Arbeitseinsatz von Strafgefangenen im Bergbau getroffen worden sei. Zu diesem Zeitpunkt habe ein Bedarf an 820 zusätzlichen Arbeitskräften im Steinkohlenbergbau bestanden, der aus dem Gesamtbestand der Strafgefangenen abgedeckt werden sollte. Bereits damals sei festgelegt worden, daß den Gefangenen zwei Arbeitstage als drei Hafttage angerechnet werden. Dieser "Leistungsanreiz" fand Eingang in die Verordnung vom 3. 4. 1952. Vgl. ebenda, Bl. $68 \mathrm{f}$.

145 Ebenda, Bl. $11 \mathrm{f}$.

146 Gesetzblatt der DDR 1952, S. $275 \mathrm{f}$.

147 BAB, DQ 2/1871, Abt. Arbeitskraftlenkung (Heisig) am 25. 2. 1952 an die Landesregierungen in Potsdam, Schwerin, Halle, Dresden und Erfurt.

$1+8$ Ebenda, Aktenvermerk der Abt. Arbeitskraftlenkung vom 15. 8. 1952, S. 1.

149 So wurde z. B. erwogen, das Eisenhüttenwerk Thale als weiteren Arbeitsschwerpunkt festzulegen. Vgl. ebenda, S. 2.

150 BAB, DQ 2/1871, Aktenvermerk der Abt. Arbeitskraftlenkung vom 22. 10. 1952. 